\title{
Production mechanism of hot nuclei in violent collisions in the Fermi energy domain.
}

\author{
M. Veselsky *†‡ \\ Cyclotron Institute, Texas A\&M University, College Station, TX 77843.
}

\begin{abstract}
A production mechanism of highly excited nuclei formed in violent collisions in the Fermi energy domain is investigated. The collision of two nuclei is decomposed into several stages which are treated separately. Simplified exciton concept is used for the description of preequilibrium emission. A modified spectator-participant scenario is used where motion along classical Coulomb trajectories is assumed. The participant and one of the spectator zones undergo incomplete fusion. Excitation energies of both cold and hot fragment are determined. Results of the calculation are compared to recent experimental data in the Fermi energy domain. Data on hot projectile-like, mid-velocity and fusion-like sources are described consistently. Geometric aspects of pre-equilibrium emission are revealed. Explanations to previously unexplained experimental phenomena are given. Energy deposited into non-thermal degrees of freedom is estimated.
\end{abstract}

PACS: 24.10.-i; 24.10.Lx; 25.70.-z; 25.70.Lm; 25.70.Mn; 25.70.Pq

Keywords: Nuclear reactions; E $=20$ - $100 \mathrm{~A} \mathrm{MeV;} \mathrm{Pre-equilibrium} \mathrm{emission;} \mathrm{Incomplete} \mathrm{fusion;}$ Multifragmentation; Collective flow

\section{Introduction}

A detailed knowledge of the production mechanism of hot nuclei is desirable for understanding the processes leading to multifragmentation. The peripheral collisions of heavy ions in the Fermi energy domain demonstrate that nucleon exchange is a dominating mechanism leading to the production of highly excited quasiprojectiles [1, 2]. In more violent collisions, the processes leading to midvelocity emission start to play an important role. The influences of pre-equilibrium emission and fragmentation-like processes become important. The hot source is created which further undergoes multifragmentation. The origin and properties of the hot mid-velocity source remain still a matter of discussions. In order to obtain properties of the hot source the experimental studies performed with large angular coverage ( preferably in $4 \pi$-geometry ) are of primary interest. Considerable amounts of such data were obtained during last decade [3, 4, 5, 6, 6, 8]. In this article, a model of production of highly excited nuclei in violent collisions based on several simple phenomenological assumptions is presented and its capability to explain trends of the experimental observables related to dynamical properties and de-excitation of the hot source created in violent collisions is investigated.

\footnotetext{
*Phone: (979)-845-1411, fax: (979)-845-1899, e-mail: veselsky@comp.tamu.edu

${ }^{\dagger}$ On leave of absence from Institute of Physics, Slovak Academy of Sciences, Bratislava, Slovakia

${ }^{\ddagger}$ Address after Apr 1, 2002: Institute of Physics, Slovak Academy of Sciences, Dubravska cesta 9, Bratislava, Slovakia, e-mail: fyzimarv@savba.sk
} 


\section{Model}

The physical picture employed in the calculations is depicted in Fig. 1. Properties of the highly excited nuclei in violent collisions are determined on an event-by-event basis in the Monte Carlo fashion. The model considers several stages of the collision. Different stages of the collision are treated separately. First, pre-equilibrium particle emission takes place. Later, the intermediate projectile-target system is reconstructed and the participant and spectator zones are determined. Finally, an incomplete fusion channel is chosen via interaction of spectators with the participant zone.

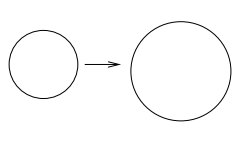

Initial stage

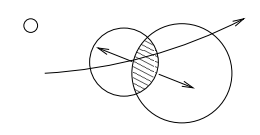

Pre-equilibrium emission

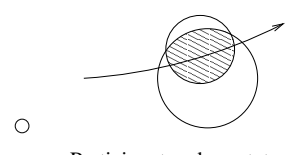

Participant and spectators

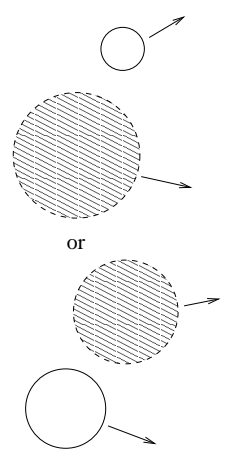

Incomplete fusion

Figure 1: Schematic description of the physical picture employed in the model calculation for violent collisions. Shaded areas mean overlapping and later hot regions.

\section{Pre-equilibrium emission}

The pre-equilibrium emission ( $\mathrm{PE}$ ) is a process where fast particles are emitted prior to the equilibration of the system. The emission of fast pre-equilibrium particles in the reactions induced by nucleons and light particles was theoretically explained using the exciton model [9]. For reactions induced by heavy-ion beams a model of nucleon exchange was developed [10]. In the present work, we use a phenomenological description [11] based on similar assumptions as the exciton model. The probability of pre-equilibrium emission for a given reaction stage is evaluated using the formula

$$
P_{p r e}\left(n / n_{e q}\right)=1-e^{-\frac{\left(n / n_{e q}-1\right)^{2}}{2 \sigma^{2}}}
$$

for $n \leq n_{e q}$ and equals zero for $n>n_{e q}$, where $n$ is the number of excitons at a given stage and $n_{e q}$ is the the number of excitons in the fully equilibrated compound system ( consisting of both projectile and target ) for a given excitation energy. The basic assumption leading to equation (11) is that $\mathrm{P}_{\text {pre }}$ depends exclusively on the ratio $n / n_{e q}$ as can be deduced from the results of ref. [12] where the density of particle-hole states is approximately described using a Gaussian centered at $n_{e q}$. The parameter $\sigma$ is a free parameter of the calculation and no dependence on excitation energy is assumed. An initial exciton number is equal to the mass number of the projectile nucleus. The equilibrium number of excitons in the fully equilibrated compound system is calculated according to the formula 12

$$
n_{e q}=2 g T \ln 2
$$

where $g$ is the single particle level density at the Fermi energy and $T$ is the nuclear temperature determined as $T^{2}=U / \tilde{a}$, where $\tilde{a}$ is the level density parameter $(\tilde{a}=A / 9)$ and $U$ is the excitation 
energy. At each emission step, a random number between zero and one is generated. If the random number is smaller than $\mathrm{P}_{\text {pre }}$ a pre-equilibrium particle is emitted. If no pre-equilibrium emission occurs at a given emission stage, the pre-equilibrium stage is finished.

The properties of emitted pre-equilibrium particles are determined for every emission. The type of particle emitted is determined randomly using the Hauser-Feschbach emission widths for neutron, proton and $\alpha$-particle. A Maxwellian spectrum of kinetic energy with an apparent temperature [13, 14]

$$
T_{\text {app }}=\left[\frac{2.5}{A_{P}}\left(E_{P}-V_{C}\right)\right]^{1 / 2}
$$

is assumed, where $A_{P}$ is the projectile mass number, $E_{P}$ is the projectile energy and $V_{C}$ is the Coulomb barrier of projectile and target. The apparent temperature corresponds to the excitation energy of the fireball formed by the projectile nucleus together with another $\mathrm{A}_{P}$ nucleons from the target nucleus. This model describes successfully experimental systematics of measured apparent temperatures as shown in refs 13, 14. Particles are emitted isotropically from the fireball frame moving at half the projectile velocity. After emission, the exciton number is increased by a value

$$
\Delta n=A_{\text {pre }} \frac{\kappa}{\beta_{0 r}(l)}
$$

where $\mathrm{A}_{\text {pre }}$ is the mass of emitted particle, $\beta_{0 r}(l)$ is the radial velocity in the contact configuration at a given angular momentum and $\kappa$ is a free parameter.

\section{Projectile and target after pre-equilibrium emission}

Since pre-equilibrium emission is assumed to occur prior to the fragmentation stage, it is necessary to reconstruct the post-pre-equilibrium projectile-target configuration. The conclusions of the work [10] imply that in the asymmetric reactions with light projectile and heavy target the preequilibrium particles are mostly emitted from the projectile and propagate through the target. In the present work we assume that the net mass loss caused by emission of pre-equilibrium particles is distributed between projectile and target according to the relation

$$
\frac{\Delta A_{P}}{\Delta A_{T}}=\frac{A_{T}}{A_{P}}
$$

which complies to the conclusions of work [10] for asymmetric systems and gives equal net mass losses for the symmetric case. For the charge, a distribution of the net charge loss similar to Eqn. (5) would lead to unbound projectile species for mass asymmetric projectile-target systems. To avoid that, a net charge loss of the projectile is chosen as

$$
\Delta Z_{P}=\Delta A_{P} \frac{Z_{P}+Z_{T}}{A_{P}+A_{T}}
$$

Since the emission from the fireball [13, 14] implies that an emitted particle undergoes typically one nucleon-nucleon collision, one can expect that the total excitation energy of the projectile and target after the pre-equilibrium stage would track with the sum of kinetic energies of the emitted pre-equilibrium particles. On the other hand, in the case when multiple pre-equilibrium particles were already emitted one should take into account the possibility that any further nucleon-nucleon collision can also lead to the decrease of the excitation energy. We employ a formula

$$
E_{\text {tot }}^{*}=\left(1+\sqrt{\Delta A_{\text {pre }}}\right) \frac{E_{k}^{\text {pre }}}{\Delta A_{\text {pre }}}
$$


where $E_{t o t}^{*}$ is the total excitation energy, $E_{k}^{\text {pre }}$ is the sum of kinetic energies of all pre-equilibrium particles and $\Delta A_{\text {pre }}$ is the net mass loss due to pre-equilibrium emission. Formula (7) is an approximation to the case where a random walk in excitation energy starts after the first two emissions. The excitation energy of the projectile and target is proportional to their masses. This is consistent with formula (5) since every particle originating from the projectile propagates through the target ( and vice versa ) where a collision occurs.

\section{Spectators and participant}

Several geometrical models of fragmentation have been proposed before where projectile and target are supposed to follow a straight trajectory determined by an impact parameter [15, 16]. Other works assume a classical Coulomb trajectory up to the closest approach configuration and only later the nuclei are supposed to follow a straight line [17, 18]. In the Fermi energy domain, where angular momentum plays an important role, the classical Coulomb trajectories are more realistic since conservation of angular momentum is assured.

In the present work, we assume a classical Coulomb trajectory of the intermediate projectiletarget system without making any additional assumptions. The minimum distance between the intermediate projectile and target is used as a principal parameter of the geometric overlap scenario. For any possible trajectory, the overlapping volume is not smaller than the overlap of two spheres at minimum distance ( closest approach ). On the other hand, the geometric overlap formula of the abrasion-ablation model [15] always gives an overlap volume for a given minimum distance larger than the one along the classical Coulomb trajectory. The exact result is between these two values. We determine the volume of the participant zone randomly from the interval with limiting values given by the abrasion-ablation formula and the two-sphere overlap formula. Such a value differs from the exact result but nevertheless can be a reasonable approximation when taking into account irregularities of the separating nuclear surfaces. As a result, one participant and one or two spectator zones are created in the fragmentation stage. Their masses are determined proportionally to the determined volumes. The charges of the spectators are determined according to the combinatorial probability density 19, 20]

$$
P\left(Z_{i S}\right)=\frac{\left(\begin{array}{c}
Z_{i} \\
Z_{i S}
\end{array}\right)\left(\begin{array}{l}
N_{i} \\
N_{i S}
\end{array}\right)}{\left(\begin{array}{c}
A_{i} \\
A_{i S}
\end{array}\right)}
$$

where $\mathrm{A}_{i}, \mathrm{Z}_{i}, \mathrm{~N}_{i}$ are the mass number, charge and neutron number of the projectile ( target ) and $\mathrm{A}_{i S}, \mathrm{Z}_{i S}, \mathrm{~N}_{i S}$ are the mass, charge and neutron number of the projectile ( target ) spectator.

\section{Incomplete fusion}

In the Fermi energy domain one can assume that the participant zone will not necessarily exist individually but can be captured by either the projectile or the target spectator zone. Especially in the symmetric reaction it is reasonable to assume that the capture by either of the spectators should be equally probable. To make a choice, for both spectators the volumes were determined within a distance of $1 \mathrm{fm}$ from the separation plane in order to estimate the number of spectator nucleon which interact with the participant nucleons via nuclear interaction. The volume was approximated by a $1 \mathrm{fm}$ thick segment of the sphere touching the participant zone in the closest approach configuration. The number of neighboring nucleons $\left(A_{N S}\right)$ is then determined using a Gaussian distribution centered at the value exactly corresponding to the volume with the standard deviation equal to $\sqrt{A_{N S}}$. The participant zone is captured by a spectator with more neighboring 
nucleons. The capturing spectator and participant zone form a hot fragment. The remaining spectator zone is much colder.

The excitation energy of the cold fragment is determined assuming that the part of the kinetic energy of the relative motion of the cold fragment and participant zone is transferred into the internal heat during the separation via collisions of the spectator and participant nucleons along the separation plane. The formula for excitation energy reads

$$
E_{S}^{*}=x A_{N S}\left(\frac{E_{P}-V_{C}}{A_{P}}\right) \frac{<s>}{\lambda}
$$

where $E_{P}$ and $A_{P}$ are the kinetic energy and the mass number of the intermediate projectile after pre-equilibrium emission, $V_{C}$ is the Coulomb barrier between the cold and hot fragment in the contact configuration and $\langle s\rangle=\frac{8\left\langle r_{s e g}\right\rangle}{3 \pi}$ is the mean path of the spectator nucleon within the touching segment of the sphere along the separation plane. For the mean free path $\lambda$ a value 6 $\mathrm{fm}$ is adopted. For each collision half of the asymptotic kinetic energy is converted into heat on average ( $x$ is a random number between zero and one ).

The kinetic energy and the emission angle of the cold fragment are determined randomly using the double differential cross section formula based on the Serber approximation [21]

$$
\frac{d^{2} \sigma}{d E_{a} d \Omega_{a}}=\frac{\left(E_{a} E_{b}\right)^{1 / 2}}{\left(2 \mu B_{P}+2 m_{a}^{2} E_{P} / m_{P}+2 m_{a} E_{a}-4\left(m_{a}^{3} E_{P} E_{a} / m_{P}\right)^{1 / 2} \cos \theta\right)^{2}}
$$

where it is the fragment $a$ which flies away and the fragment $b$ which fuses with the other nucleus, $E_{a}$ and $E_{b}$ are their c.m. kinetic energies, $B_{P}$ is the binding energy of $a$ and $b$ in $P, \mu$ is the reduced mass of the system $a+b, m_{P}, m_{a}, m_{b}$ are the masses of $P, a, b$ and $\theta$ is the emission angle of $a$ with respect to the direction of $P$ in the closest approach.

In the case where the cold fragment originates from the target, the system is transformed into the inverse frame, formulas (9) and (10) are used and the system is transformed back into the normal frame. The excitation energy, kinetic energy and the angle of a hot fragment are determined from the kinematics. The intrinsic angular momentum of the hot fragment is calculated using a mean radial distance and momentum of the participant zone relative to the capturing spectator in the closest approach configuration. An orbital angular momentum is determined from the relative motion of the cold and hot fragment in the contact configuration and the intrinsic angular momentum of the cold fragment is determined assuming conservation of the total angular momentum.

\section{Results and discussion}

In order to investigate the proposed model we performed an extensive comparison of the results of model calculation for different reaction stages to available ( mostly not explained satisfactorily by models used in original works ) data obtained in the Fermi energy domain. Since the experimental data usually contain the events originating from both peripheral and violent collisions, the calculation was carried out ( unless specified otherwise) for the angular momentum range from zero to grazing angular momentum. The number of events per partial wave was proportional to the angular momentum. No ad hoc criterium distinguishing between peripheral and violent collisions was implemented. Instead, for each event the Monte Carlo deep-inelastic transfer ( DIT ) code of Tassan-Got [1] was used after pre-equilibrium stage. There it is assumed that a di-nuclear configuration is created only when the overlap of nuclei does not exceed $3 \mathrm{fm}$. An excited quasi-projectile and quasi-target were created in such cases and de-excitation followed. When the overlap exceeded 
$3 \mathrm{fm}$, the collision was considered violent and a spectator-participant concept was implemented. For the reactions in inverse kinematics, namely when the projectile is heavier than the target, the system was transformed into the inverted frame where the projectile becomes a target and vice versa. Then the calculation proceeded as described above and the final kinematic properties of the reaction products were obtained after a transformation back into the laboratory frame.

\section{Multiplicities of pre-equilibrium particles}

The model of pre-equilibrium emission was compared to the results of work [22]. There a multiplicity of the pre-equilibrium particles was determined in coincidence with the projectile-like fragments ( PLF ) in the reactions of Ca beams with ${ }^{112} \mathrm{Sn}$ target at $35 \mathrm{~A} \mathrm{MeV}$. The measured multiplicities have been found significantly higher than predictions by nucleon exchange model ( NEM ) [10].

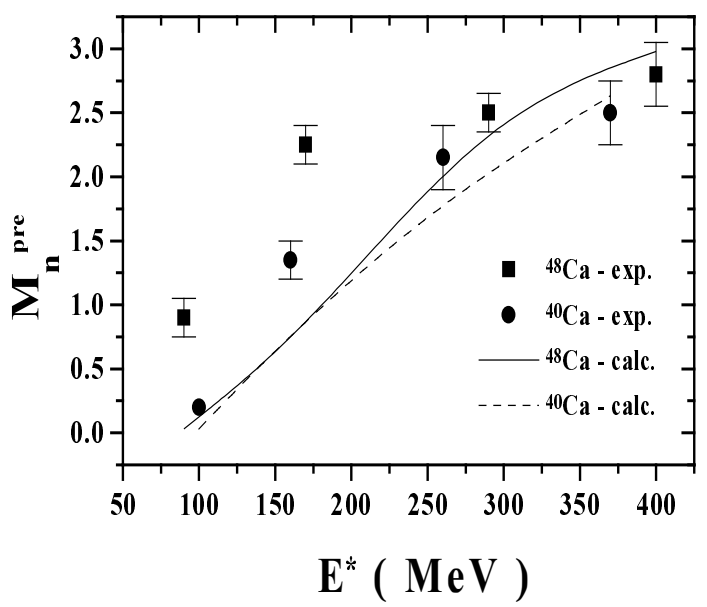

Figure 2: Experimental 22] and calculated mean multiplicities of pre-equilibrium neutrons as a function of total excitation energy. Solid squares - experimental multiplicities measured in reaction of $35 \mathrm{~A} \mathrm{MeV}{ }^{48} \mathrm{Ca}$ beam with ${ }^{112} \mathrm{Sn}$ target, solid circles - ditto for $35 \mathrm{~A} \mathrm{MeV}{ }^{40} \mathrm{Ca}$ beam, solid line - calculated multiplicities in reaction of 35 $\mathrm{A} \mathrm{MeV}{ }^{48} \mathrm{Ca}$ beam with ${ }^{112} \mathrm{Sn}$ target, dashed line - ditto for $35 \mathrm{~A} \mathrm{MeV}{ }^{40} \mathrm{Ca}$ beam.

Fig. 目 gives the values of the pre-equilibrium neutron multiplicity in the reactions ${ }^{40,48} \mathrm{Ca}+{ }^{112} \mathrm{Sn}$ for several bins of the total excitation energy. The solid squares and circles represent the results of work [22] and the lines represent the results of the calculation. There are some differences for the bins with lower excitation energies ( especially for ${ }^{48} \mathrm{Ca}+{ }^{112} \mathrm{Sn}$ ) which can be caused by inconsistency of experimentally determined and calculated excitation energies for the peripheral collisions. For more central bins the agreement is quite good. The parameters $\sigma=0.22$ and $\kappa=0.3$ were used in the calculation. The same values of $\sigma$ and $\kappa$ were used in other reactions and lead to results which track well with the results of experimental works where multiplicities of preequilibrium particles were determined ( or at least estimated ) in coincidence with the heavy residues or fission fragments [23, 24, 11] or in coincidence with the reconstructed quasi-projectile [2].

Fig. 3 gives multiplicities of pre-equilibrium neutrons for the reaction ${ }^{20} \mathrm{Ne}+{ }^{165} \mathrm{Ho}$ at the projectile energies $11-30 \mathrm{~A} \mathrm{MeV} \mathrm{[23,} \mathrm{24].} \mathrm{The} \mathrm{squares} \mathrm{represent} \mathrm{the} \mathrm{experimental} \mathrm{data} \mathrm{and}$ the line represents the calculation using parameters $\sigma=0.22$ and $\kappa=0.3$. The overall agreement is reasonable. Also for this data, the NEM model [10] was used and underestimated the experimental data ( a note added in proof in the original article [10] suggests possibility to improve the agreement between experiment and calculation ). The work [11] estimates the probability value of 0.3 for the 


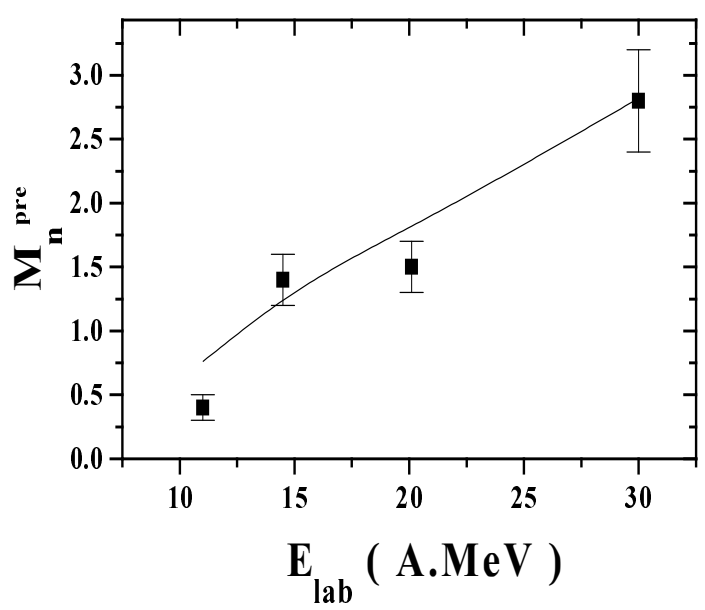

Figure 3: Experimental 23, 24] and calculated mean multiplicities of pre-equilibrium neutrons as a function of kinetic energy of the projectile. Solid squares - experimental multiplicities measured in reactions of $11-30 \mathrm{~A} \mathrm{MeV}$ ${ }^{20} \mathrm{Ne}$ beam with ${ }^{165}$ Ho target, solid line - calculated neutron multiplicities for the same reaction.

emission of four pre-equilibrium nucleons in coincidence with evaporation residues with $\mathrm{Z} \geq 87$ in the reaction ${ }^{20} \mathrm{Ne}+{ }^{208} \mathrm{~Pb}$ at projectile energy $15 \mathrm{~A} \mathrm{MeV}$. For the emission of six pre-equilibrium particles the probability value 0.1 is given. Corresponding calculated values $(1 \leq 50)$ are 0.25 and 0.07. Thus the calculation describes reasonably the multiplicity of pre-equilibrium particles in the central collisions at projectile energy $15 \mathrm{~A} \mathrm{MeV}$. The multiplicity of pre-equilibrium protons was estimated in the reaction ${ }^{28} \mathrm{Si}+{ }^{112} \mathrm{Sn}$ for peripheral collisions in coincidence with the reconstructed hot projectile-like fragments [2]. The experimental estimates of proton multiplicities 0.2 for 30 $\mathrm{A} \mathrm{MeV}$ and 0.3 for $50 \mathrm{~A} \mathrm{MeV}$ correspond well to the calculated values 0.25 and 0.34 , respectively. The calculated values were obtained using the model of pre-equilibrium stage, the DIT code [1] and the filtering procedure. The comparisons show that given description of multiplicities of the pre-equilibrium particles is consistent with the experimental trends. We adopt the parameters $\sigma=0.22$ and $\kappa=0.3$ as a standard set for further studies.

Based on the comparisons of the calculation to experimental data one can try to understand the physical essence of the parameters $\sigma$ and $\kappa$ and to make conclusions concerning the nature of the process of pre-equilibrium emission. The parameter $\sigma$ can be, in principle, related to the width of the distribution of the particle-hole states which peaks at $n_{e q}$ and can be approximated by a Gaussian. The value $\sigma=0.22$ is about three time larger than the variance of the distribution of particle-hole states calculated using the formula given in [12] for the range of the masses and excitation energies corresponding to the reactions considered here. Since the assumptions made in [12] are valid only for moderate excitations, a larger value of $\sigma$ suggests that either the distribution of the particle-hole states at high excitations is wider than the distribution calculated using formula from [12] or a larger value of $\sigma$ means that $n_{e q}$ grows slower with excitation energy than predicted in [12]. Compared to the value $\sigma=0.3$ in the original paper [11] the value $\sigma=0.22$ means decrease of approximately $30 \%$. The increase in the probability of pre-equilibrium emission is counterbalanced by angular momentum profile of $\Delta n$ causing decrease of pre-equilibrium multiplicity at large angular momenta ( in the original paper no angular momentum dependence of $\Delta n$ was assumed ). The value of $\kappa=0.3$ suggests that, in the given range of radial velocities, the exciton number increases typically by 2 4 excitons between two subsequent pre-equilibrium emissions. This implies that particle emissions follow each other rather quickly since the exciton number increase between emissions is comparable 
with the minimum step of the exciton model ( $\Delta n=2$ ) towards equilibrium.

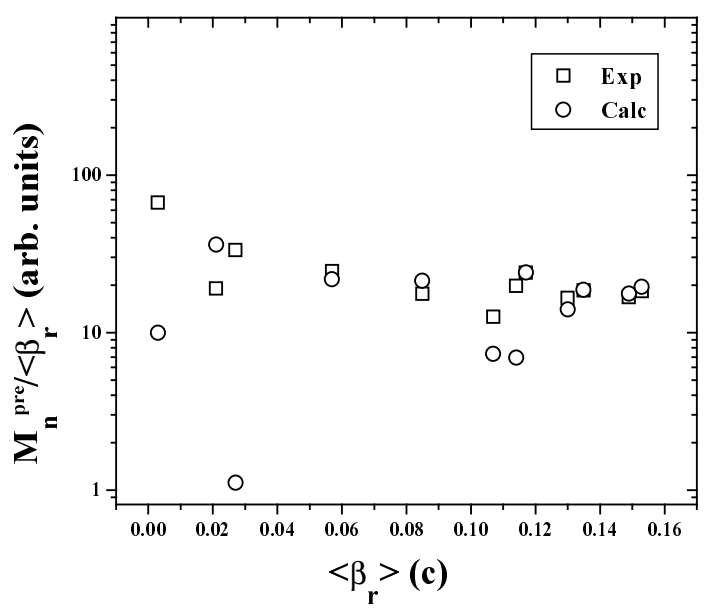

Figure 4: A dependence of the ratio of the mean multiplicity of pre-equilibrium neutrons to the mean radial velocity as a function of the latter. Experimental 22, 23, 24] ( squares ) and calculated ( circles ) multiplicities have been used.

As follows from the relation (4) the average rate of emitted pre-equilibrium particles per exciton number step during the collision $(1 / \Delta n)$ can be considered proportional to the radial velocity in the contact configuration $\beta_{0 r}(l)$ for a given angular momentum. Since $\beta_{0 r}(l)$ is an initial radial velocity it would be more appropriate to relate the emission rate to a mean radial velocity during

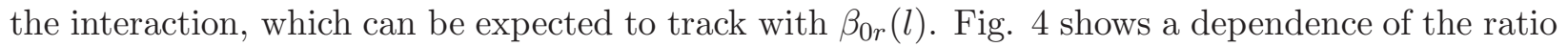
of the mean multiplicity of pre-equilibrium neutrons to the mean radial velocity as a function of the latter. The mean radial velocity was calculated for a given angular momentum range along the classical Coulomb trajectories from the contact configuration to the point where $\beta_{r}$ drops under the threshold value $4 \mathrm{~A} \mathrm{MeV}$. The data from the Fig. 2 and 3 are used in Fig. 4 For the data from Fig. 2, the calculated angular momentum ranges corresponding to the excitation energy bins were used. For the data from Fig. 3, the angular momentum range from zero to grazing angular

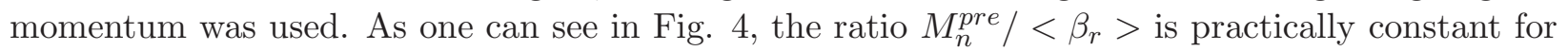
experimental ( squares ) and with few exceptions also for calculated ( circles ) multiplicities. Thus, the mean multiplicity of pre-equilibrium neutrons ( and particles in general ) is proportional to a mean radial velocity $\left\langle\beta_{r}>\right.$ determined for a corresponding range of angular momenta along the classical Coulomb trajectories.

The multiplicity of pre-equilibrium particles for a given collision can be expressed as radial integral

$$
M^{\text {pre }}=\int d r \frac{d M^{\text {pre }}}{d n} \frac{d n}{d r},
$$

where $r$ is the distance from the center of the target nucleus at a given point along the classical Coulomb trajectory. The exciton number change per radial distance $\frac{d n}{d r}$ can be related to the nucleon-nucleon cross section via $\frac{d n}{d l}$ which represents an exciton number change per unit distance along the trajectory. When assuming that the number of excitons increases as a result of two-body collisions, the observable $\frac{d n}{d l}$ can be directly related to the nucleon-nucleon cross section and can be considered proportional to $1 / \beta$. Then $\frac{d n}{d r}$ becomes proportional to $1 / \beta_{r}$. The validity of the relation (4) can be extended to any moment of the reaction and the emission rate $\frac{d M^{p r e}}{d n}$ can be considered 


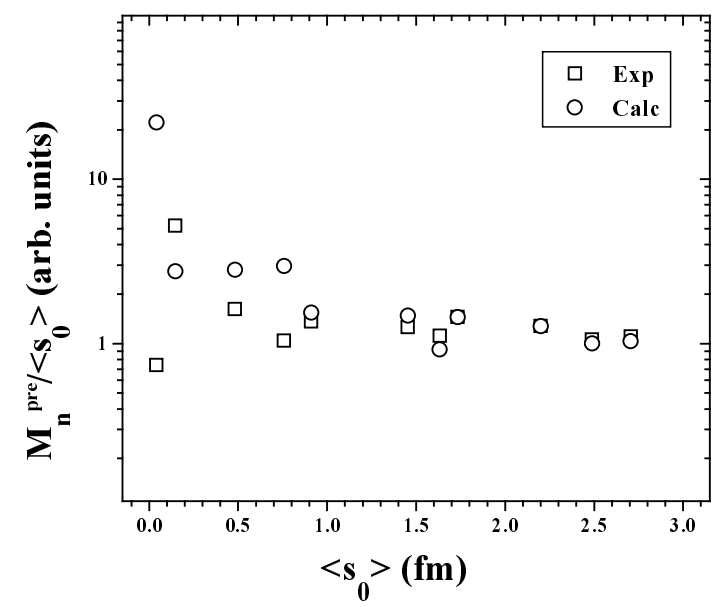

Figure 5: A dependence of the ratio of the mean multiplicity of pre-equilibrium neutrons to the mean overlap $M_{n}^{\text {pre }} /<s_{0}>$ as a function of $\left\langle s_{0}>\right.$. Experimental 22, 23, 24 ( squares ) and calculated ( circles ) multiplicities have been used.

proportional to $\beta_{r}$. Then, the integrand in (11) becomes a constant and the mean multiplicity of pre-equilibrium particles should be also proportional to the mean value of radial overlap $\left\langle s_{0}\right\rangle$ where radial velocity drops below the threshold value $4 \mathrm{~A} \mathrm{MeV}$ which can be expected to track with $\left\langle\beta_{r}\right\rangle$. In Fig. 5 we show a dependence of the ratio of multiplicity of pre-equilibrium neutrons to the mean overlap $M_{n}^{\text {pre }} /<s_{0}>$ as a function of $<s_{0}>$. Indeed, one can see that for the mean overlap larger than $0.8 \mathrm{fm}$ the ratio is again practically constant for both experimental ( squares ) and calculated ( circles ) multiplicities ( experimental values are practically constant from $0.3 \mathrm{fm}$ above ). Thus, the picture given by equation (11) can be considered consistent with the essential features of the process.

When making conclusions concerning Figs. 1 and 5 , caution is necessary since no deceleration by the recoil from the emitted particles is considered. Nevertheless, we assume that even then the picture will not change dramatically. Then, taking into account the comparison of experimental data on multiplicities of the pre-equilibrium particles with the results of model calculations and the results of following geometric analysis, the process of pre-equilibrium emission can be qualitatively understood as a process of two-body dissipation along the classical Coulomb trajectory where the nucleons scattered in radial direction are emitted. In this picture, the pre-equilibrium stage stops when the radial motion disappears and the relative motion of the projectile and target is mostly tangential. When the tangential motion is slow enough, friction force can transform it into rotation of a di-nuclear system as in deep-inelastic collisions. When the tangential motion is fast, a violent collision follows and formation of the participant and spectator zones along the classical Coulomb trajectory appears to be a natural next stage of the collision.

\section{Projectile-like fragments}

In the recent experimental work [25] a linear correlation between the primary mass of the projectilelike fragment and the net mass loss due to the de-excitation was reported in the nearly symmetric reactions of ${ }^{93} \mathrm{Nb}$ with ${ }^{116} \mathrm{Sn}$ at $25 \mathrm{~A} \mathrm{MeV}$ in both normal and inverse kinematics for different dissipation bins. The net mass loss increases with the primary mass of the projectile-like fragment. With increasing dissipation this trend occurs in the still broader range of primary masses. Since 
the net mass loss is correlated to the excitation energy of the hot primary projectile-like nucleus, one can expect a similar trend for the excitation energy. It was demonstrated that both rapid growth of mass variance and asymmetric excitation energy sharing can not be explained within the concept of nucleon exchange through window between nuclei ( the NEM code [26] was used in the analysis ). As an explanation, a dynamical scenario as a neck rupture was suggested.

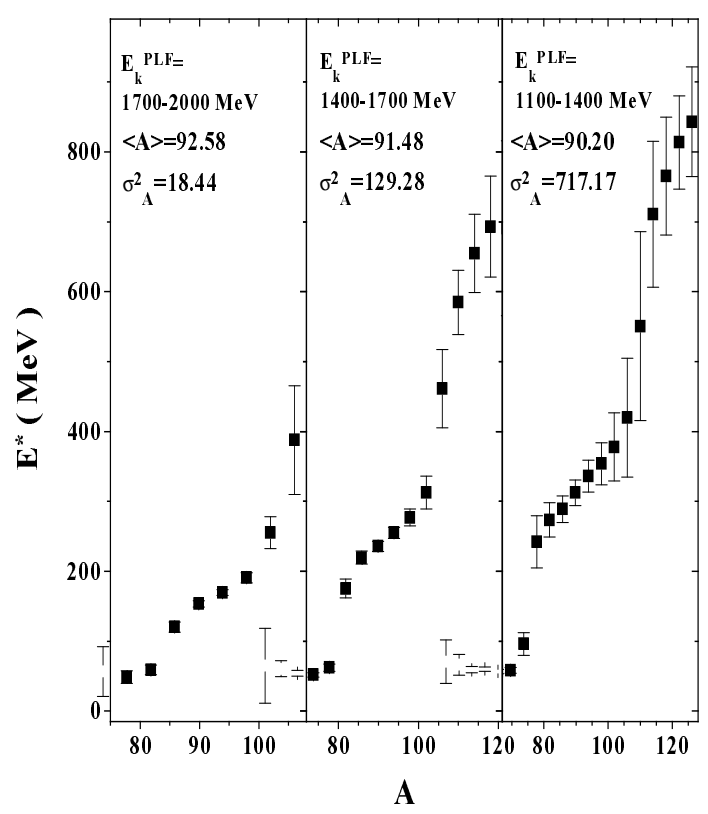

Figure 6: Calculated correlation between the primary mass and the excitation energy of a projectile-like fragment in the reaction ${ }^{93} \mathrm{Nb}$ with ${ }^{116} \mathrm{Sn}$ at $25 \mathrm{~A} \mathrm{MeV}$.

Fig. 6 gives a calculated correlation between the excitation energy and the mass of the hot projectile-like nucleus for different bins of kinetic energy. The calculation mimiques the experimental trend of the emitted mass ( a measure of excitation energy ) shown in Fig. 10 of the experimental article [25]. At masses close to the beam the deep-inelastic transfer takes place but the range of primary masses is limited. To achieve larger mass changes more violent collision should occur. When the target strips a part of the projectile, the projectile-like fragment remains relatively cold. Hot projectile-like fragments are produced if a part of the target is picked-up by the projectile. For DIT events the mean excitation energy per nucleon is practically constant what leads to a slight increase of excitation energy with increasing mass. At the region where the violent collisions start to dominate a rapid change in excitation energy per nucleon takes place. The calculated correlations deviate from straight lines in the transition regions between deep-inelastic and incomplete fusion scenario. This discrepancy can be caused by a sharp cutoff value of the overlap implemented in the DIT code. It could be possibly improved by employing a diffuse cutoff but this is beyond the scope of this article. Also in Fig. 6 are given mean masses and mass variances of the projectile-like fragment for given bins of kinetic energy. While the mean mass remains roughly constant the mass variance grows rapidly. The values of mass variance $\sigma_{A}^{2}$ are comparable to the values given in Fig. 19 of the experimental paper [25]. The incomplete fusion appears to describe correctly both large mass variances and asymmetric excitation energy sharing and offers viable explanation of anomalies reported in 25.

The production of heavy residues in the reaction ${ }^{197} \mathrm{Au}+{ }^{n a t} \mathrm{Ti}$ was measured recently in the inverse kinematics by Souliotis et al. [27] at the projectile energy $20 \mathrm{~A} \mathrm{MeV}$. Fig. [0 shows the 


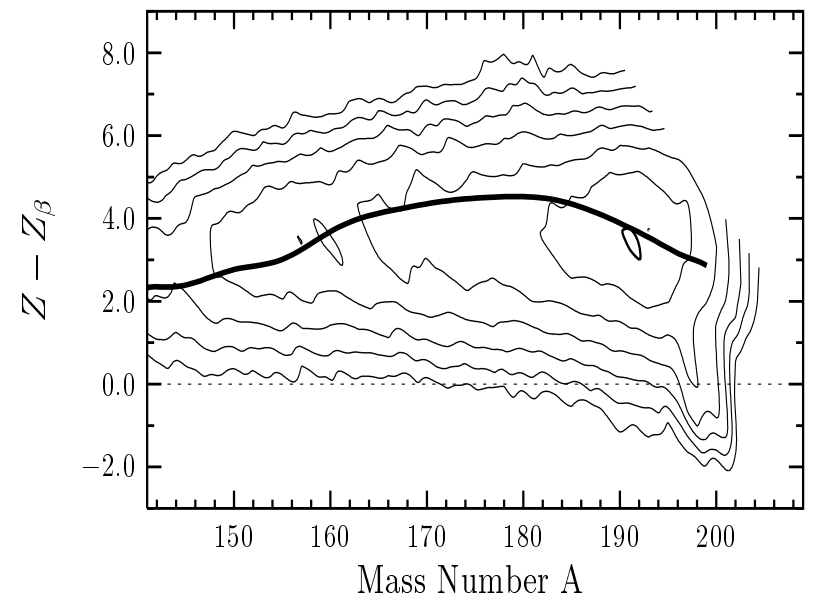

Figure 7: Measured yields [27] of heavy residues at the forward angles in the reaction ${ }^{197} \mathrm{Au}(20 \mathrm{~A} \mathrm{MeV})+{ }^{\text {nat }} \mathrm{Ti}$ as a function of $\mathrm{A}$ and $\mathrm{Z}$. Z is expressed relative to the line of $\beta$-stability. Solid line - calculated centroids of the fragment charge for given residue mass ( the code GEMINI [29] was used for de-excitation ).

measured yields of heavy evaporation residues at the forward angles as a function of $\mathrm{A}$ and Z ( $\mathrm{Z}$ is represented relative to the line of $\beta$-stability ). The experimental work [27 gives comparison to relativistic fragmentation code [28] which reproduces reasonably the charge centroids for masses far from the beam even if the validity of such approach at $20 \mathrm{~A} \mathrm{MeV}$ is questionable. The solid line in Fig. 7 represents the centroids of $\mathrm{Z}$ for a given residue mass calculated in the present work. The angle and momentum cuts corresponding to the angle and momentum acceptance of the spectrometer were applied and the statistical code GEMINI [29] was used for the de-excitation stage. One can see that the calculated centroids follow the experimental trend quite well. Thus it offers an explanation of the data appropriate for this projectile energy.

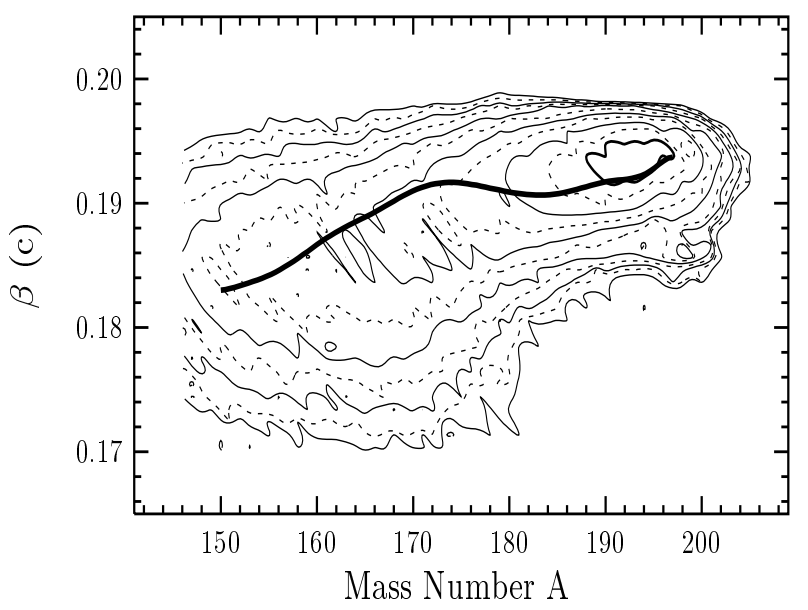

Figure 8: Experimental velocity distributions 27] of the heavy residues measured at the forward angles in the reaction ${ }^{197} \mathrm{Au}(20 \mathrm{~A} \mathrm{MeV})+{ }^{\text {nat }} \mathrm{Ti}$ plotted as a function of A. Solid line - calculated velocity centroids for given residue mass.

A dominant mechanism contributing to the production of heavy residues can be understood from Fig. 8 where the measured velocity distributions are given as a function of fragment mass. 
With decreasing mass the reaction products become still slower. Again, the line represents the calculated centroids after the spectrometer cut. One can see that the calculation follows the experimental data. A typical contributing scenario in the calculation is the emission of up to three pre-equilibrium neutrons followed by deep-inelastic transfer and by de-excitation of the heavy fragment ( the excitation energies do not exceed $3 \mathrm{~A} \mathrm{MeV}$ ). A similar agreement was obtained for the evaporation residues in the more symmetric reaction ${ }^{197} \mathrm{Au}(20 \mathrm{~A} \mathrm{MeV})+{ }^{90} \mathrm{Zr}[30$. The calculation seems to be able to provide adequate treatment of the pre-equilibrium stage and offers a natural extension of the original DIT code of Tassan-Got [1].

\section{Reaction dynamics and properties of the hot source}

The mass and excitation energy of the heavy fusion-like source have been determined in the work [31] ( the former indirectly using coalescence analysis and QMD simulation 32] and the latter using three-source fit and light charged particle ( LCP ) calorimetry techniques ) in four projectile-target combinations with the same projectile energy $47 \mathrm{~A} \mathrm{MeV}$. The experiment provided practically full coverage for LCPs at angles beyond $20^{\circ}$. Fig. 9 gives the calculated distributions of excitation energy versus mass of the heavy source for reactions ${ }^{12} \mathrm{C}+{ }^{116} \mathrm{Sn},{ }^{20} \mathrm{Ne}+{ }^{108} \mathrm{Ag},{ }^{40} \mathrm{Ar}+{ }^{100} \mathrm{Mo}$ and ${ }^{64} \mathrm{Zn}+{ }^{89} \mathrm{Y}$ at the projectile energy $47 \mathrm{~A} \mathrm{MeV}$. Only the most central events have been included into calculation. The limiting angular momentum was set so that the partial waves included represent $10 \%$ of the reaction cross section. Black squares in all plots correspond to the data from [31. In the case of the reaction ${ }^{12} \mathrm{C}+{ }^{116} \mathrm{Sn}$ the target-like nuclei with the excitation energy not exceeding $2 \mathrm{~A} \mathrm{MeV}$ do not contribute to the experimental data because of the low multiplicity of charged particles and the data point can be related only to the part of the calculated distribution above $2 \mathrm{~A} \mathrm{MeV}$. For the reaction ${ }^{20} \mathrm{Ne}+{ }^{108} \mathrm{Ag}$ the data point is located slightly away from the central part of the calculated distribution but both are still roughly consistent within experimental uncertainties. In the reactions ${ }^{40} \mathrm{Ar}+{ }^{100} \mathrm{Mo}$ and ${ }^{64} \mathrm{Zn}+{ }^{89} \mathrm{Y}$ the data point is shifted from the center of the calculated distributions towards lower excitation energies and masses. This effect seems to increase with increasing symmetry of the projectile-target combination. For the reaction ${ }^{40} \mathrm{Ar}+{ }^{100} \mathrm{Mo}$ the data point is moved slightly towards lower mass and excitation energy but still remains close to the center of distribution. For the reaction ${ }^{64} \mathrm{Zn}+{ }^{89} \mathrm{Y}$ the shift is already significant. A possible explanation of the shift can be the presence of an additional effect not included in the calculation such as collective flow which would lead to hardening of the spectra of transverse energies and thus to overestimation of direct emission within the three-source fit method and to underestimation of source excitation energy. Due to geometric coverage the three-source analysis as a model dependent technique was performed at angles $20^{\circ}$ and larger in the laboratory frame which for the increasingly symmetric reactions correspond to even larger angles in the center of mass frame and an increasing amount of information from forward angles is missing. This puts less constraint onto the properties of projectile-like source in the fitting procedure and the emission multiplicity can be overestimated. More detailed data of the same type including also the forward angles would be of interest for further study.

The reaction ${ }^{40} \mathrm{Ar}+{ }^{58} \mathrm{Ni}$ at $95 \mathrm{~A} \mathrm{MeV}$ was studied experimentally using the INDRA $4 \pi$ setup [3]. The properties of the projectile-like source have been reconstructed using two different types of analysis. In the two-source analysis, the projectile-like source consisted of all the detected particles with parallel velocity larger than the center of mass velocity. The three-source analysis employs the usual three-source fit technique. Experimental masses and excitation energies of the projectile-like source are given in Fig. 10 as a function of the impact parameter ( solid squares -3 sources, solid circles - 2 sources ). Open symbols represent the results of the calculations. As an equivalent of the three-source analysis the properties of the calculated projectile-like source are shown ( open 

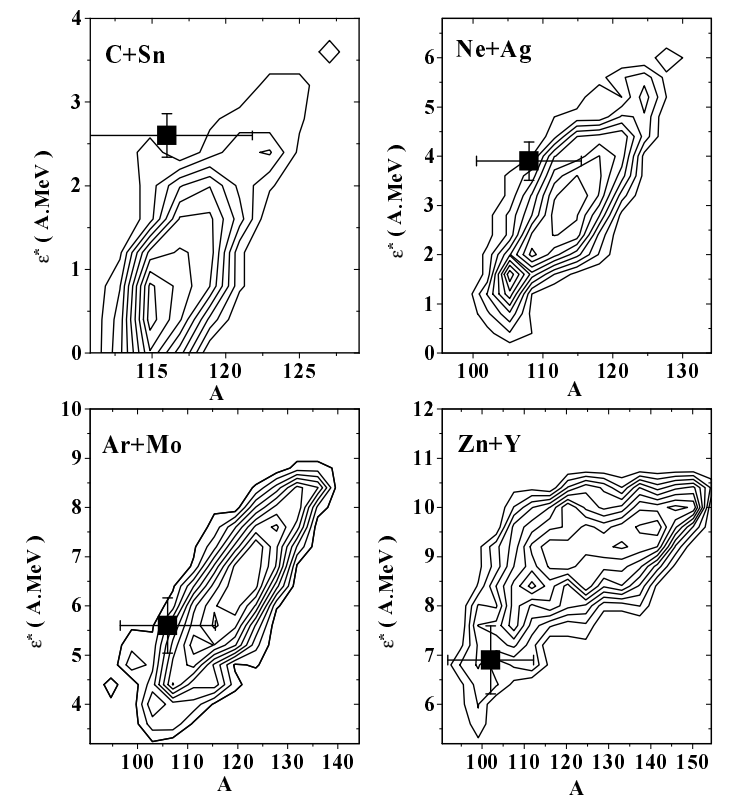

Figure 9: Calculated correlation of the excitation energy and the mass of the hot target-like sources produced in the reactions ${ }^{12} \mathrm{C}+{ }^{116} \mathrm{Sn},{ }^{20} \mathrm{Ne}+{ }^{108} \mathrm{Ag},{ }^{40} \mathrm{Ar}+{ }^{100} \mathrm{Mo}$ and ${ }^{64} \mathrm{Zn}+{ }^{89} \mathrm{Y}$ at projectile energy $47 \mathrm{~A} \mathrm{MeV}$. Solid squares show values from [31].

squares ). The two-source analysis was approximated by assuming Gaussian shapes of the both projectile- and target-like source with the width of $17 \%$ of the projectile rapidity as obtained in the experimental work [3]. The properties of the projectile-like source ( open circles ) have been obtained by an integration of the relative part of the target- and projectile-like source at velocities above the center of mass velocity. The calculated dependencies appear to follow the experimental ones reasonably well ( with some discrepancies in the transition from peripheral to violent collisions similar to Fig. 6 ). In the two-source analysis, the mass of the hot source is slightly overpredicted for most central collisions while obtaining excellent agreement in excitation energy. The properties obtained for the projectile-like source in the most central collisions can be identified with the properties of the forward half of the very hot composite mid-velocity source created by an incomplete fusion of the participant and spectator zone. The velocity of the composite source comes close to the center of mass velocity. In the three-source analysis, the mass of the projectile-like source in the most central collisions is underestimated but the experimental trend is reproduced better than using the geometrical calculation [16] presented in the experimental paper. The excitation energy in most central collisions is again underestimated. In this case, the experimental mass of about 10 with excitation energy $8 \mathrm{~A} \mathrm{MeV}$ implies that mostly nucleons and light charged particles are observed. The question is to what extent the most energetic pre-equilibrium particles contribute to this source.

The measurement of the production of intermediate mass fragments (IMFs ) in symmetric collisions ${ }^{58} \mathrm{Fe},{ }^{58} \mathrm{Ni}+{ }^{58} \mathrm{Fe},{ }^{58} \mathrm{Ni}$ at $30 \mathrm{~A} \mathrm{MeV}$ [33] determined three different sources of IMFs, the moderately excited projectile(target)-like source at velocities close to the projectile (target) and the highly excited source at velocities close to the center of mass velocity. Fig. 11 shows a calculated correlation between the excitation energy and the velocity of the hot source in the laboratory frame calculated for the reaction ${ }^{58} \mathrm{Ni}+{ }^{58} \mathrm{Ni}$ at $30 \mathrm{~A} \mathrm{MeV}$. Both projectile-like and target-like nuclei are included in the plot. One can identify three sources analogous to the ones seen in the experiment 


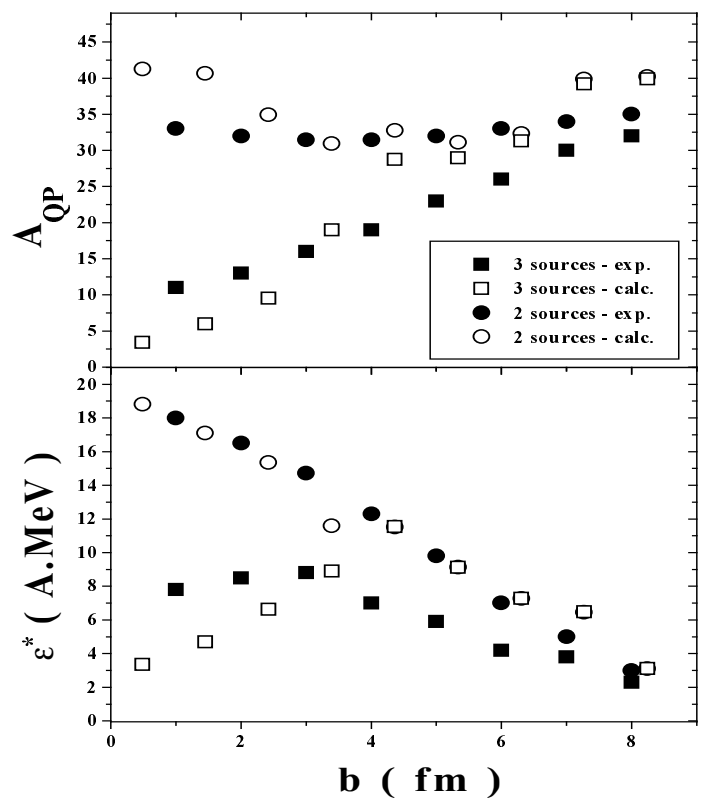

Figure 10: Experimental 33 and calculated masses and excitation energies of the projectile-like source as a function of the impact parameter for the reaction ${ }^{40} \mathrm{Ar}+{ }^{58} \mathrm{Ni}$ at $95 \mathrm{~A} \mathrm{MeV}$. Solid squares - experiment, 3 sources, solid circles - experiment, 2 sources, open squares - calculation, 3 sources, open circles - calculation, 2 sources.

in the same reaction. The projectile- and target-like sources are moderately excited. The third source with the average velocity close to the mid-velocity is highly excited. Both the projectileand target-like nuclei contribute to this source.

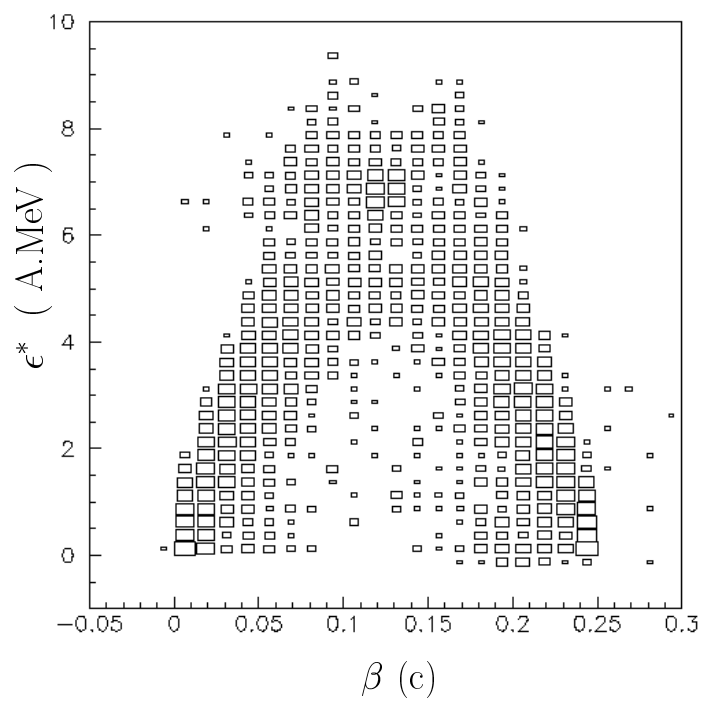

Figure 11: Calculated correlation of the excitation energy and velocity of the hot nuclei produced in the reaction ${ }^{58} \mathrm{Ni}+{ }^{58} \mathrm{Ni}$ at $30 \mathrm{~A} \mathrm{MeV}$.

In order to study this reaction in more detail, the de-excitation of hot fragments was simulated using the statistical SMM code [34]. Both projectile- and target-like source have been de-excited. 
Each source was de-excited separately and no influence of Coulomb field of the other fragment was assumed. As one can see on Fig. 12 the calculation is able to reproduce the experimentally observed fragment energy spectra at laboratory angle $40^{\circ}$ for the same reaction. Experimental spectra are depicted by thick solid lines approximating the experimental data points, calculation is represented by histograms. The normalization was chosen in order to reproduce approximately the shape and the sum of the spectra for $\mathrm{Z}=3$ at energies above $25 \mathrm{MeV}$. The shapes of spectra are reproduced satisfactory. Compared to QMD-SMM simulation the present calculation appears to reproduce better the low energy part below $50 \mathrm{MeV}$ while performing comparably in the high energy part but this may be to some extent just the effect of different normalizations.

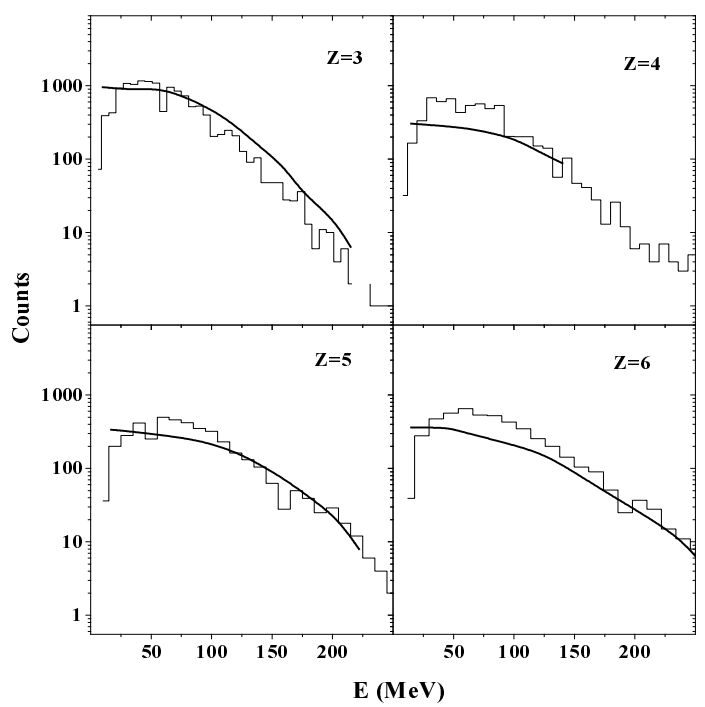

Figure 12: Kinetic energy spectra of emitted fragments with $\mathrm{Z}=3-6$ at laboratory angle $40^{\circ}$ for the reaction ${ }^{58} \mathrm{Fe}+{ }^{58} \mathrm{Ni}$ at $30 \mathrm{~A} \mathrm{MeV}$. Experimental 33] spectra are depicted by thick solid lines approximating the experimental data points, calculation is represented by histograms.

In Fig. 13 the fragment charge distributions at different angles are given. Solid squares give the experimental charge distributions at $11^{\circ}, 40^{\circ}$ and $68^{\circ}$ and histograms represent the calculation. Calculated distributions have been filtered using the energy range of the Si-Si telescopes used. Furthermore, correction for solid angle coverage of detectors was made. As one can see the calculation again performs reasonably well. At $11^{\circ}$ the experimental charge distribution is reproduced well for $\mathrm{Z}=8-15$. For $\mathrm{Z}=3-7$ calculation the experimental yields are overpredicted. According to the experimental paper [33], for the same angle the QMD-SMM calculation underpredicted yields at Z $=8-15$ while still overpredicting yields at $\mathrm{Z}=3-7$. Another calculation used in the experimental paper BUU-SMM largely overpredicted yields of lighter fragments while still underpredicting the yields of heavier fragments. Thus at forward angles the present calculation leads to better agreement than the calculations given in the original work. The reduction of the yields of fragments with $\mathrm{Z}=3-7$ can be possibly explained by an influence of Coulomb field of the heavy projectile-like fragment. At larger angles the present calculation also performs comparably ( QMD-SMM ) or better than calculations given in the experimental paper.

Furthermore, the experimental work [33] gives measured values of several isobaric and isotopic ratios at different angles. In Fig. 14a,b are given the values of experimental and calculated isobaric and isotopic yield ratios for the reaction ${ }^{58} \mathrm{Fe}+{ }^{58} \mathrm{Ni}$ at $30 \mathrm{~A} \mathrm{MeV}$ as a function of the difference of binding energies ( squares and circles - experimental values at $11^{\circ}$ and $40^{\circ}$ respectively, solid 


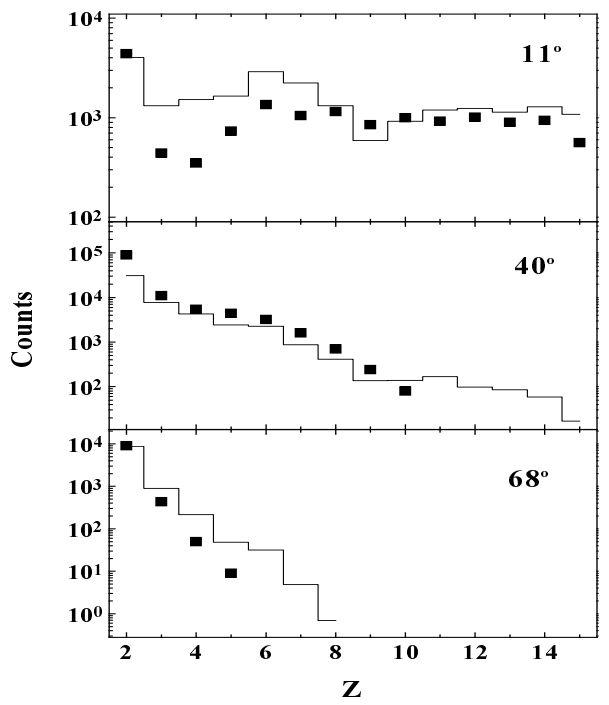

Figure 13: Fragment charge distributions at different angles for the reaction ${ }^{58} \mathrm{Fe}+{ }^{58} \mathrm{Ni}$ at $30 \mathrm{~A} \mathrm{MeV}$. Solid squares give the experimental [33] charge distributions at $11^{\circ}, 40^{\circ}$ and $68^{\circ}$ and histograms represent the calculation.
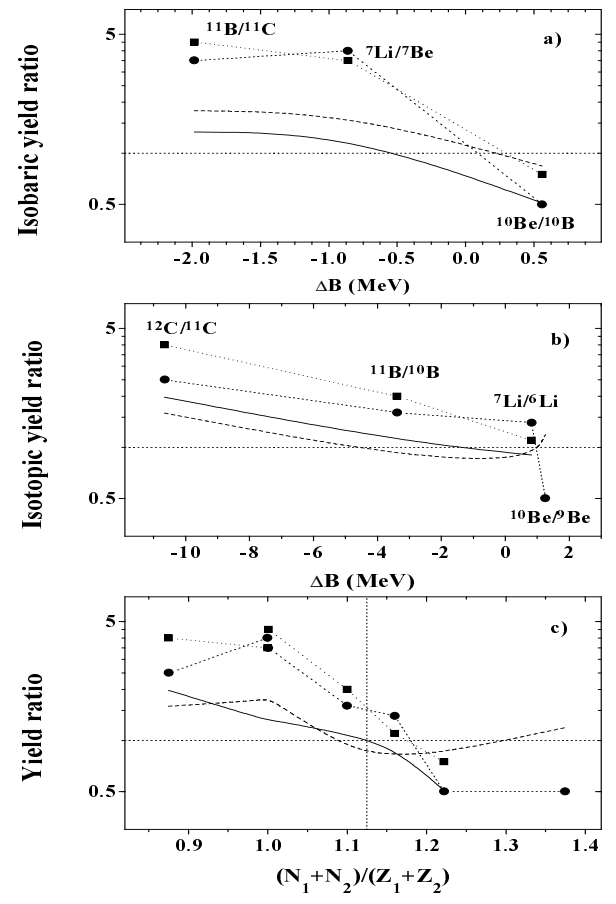

Figure 14: (a),(b) - Experimental [33] and calculated isobaric and isotopic yield ratios for the reaction ${ }^{58} \mathrm{Fe}+{ }^{58} \mathrm{Ni}$ at $30 \mathrm{~A} \mathrm{MeV}$ as a function of the difference of binding energies ( squares and circles - experimental values at $11^{\circ}$ and $40^{\circ}$ respectively, solid and dashed line - calculated values at $11^{\circ}$ and $40^{\circ}$ respectively, horizontal dotted line - unity ). (c) - Fragment yield ratios from (a),(b) plotted against average N/Z-ratio of the fragment pair ( symbols and lines analogous to (a),(b), vertical dotted line shows average $\mathrm{N} / \mathrm{Z}$-ratio of stable isotopes with $\mathrm{Z}=3-6$ ). 

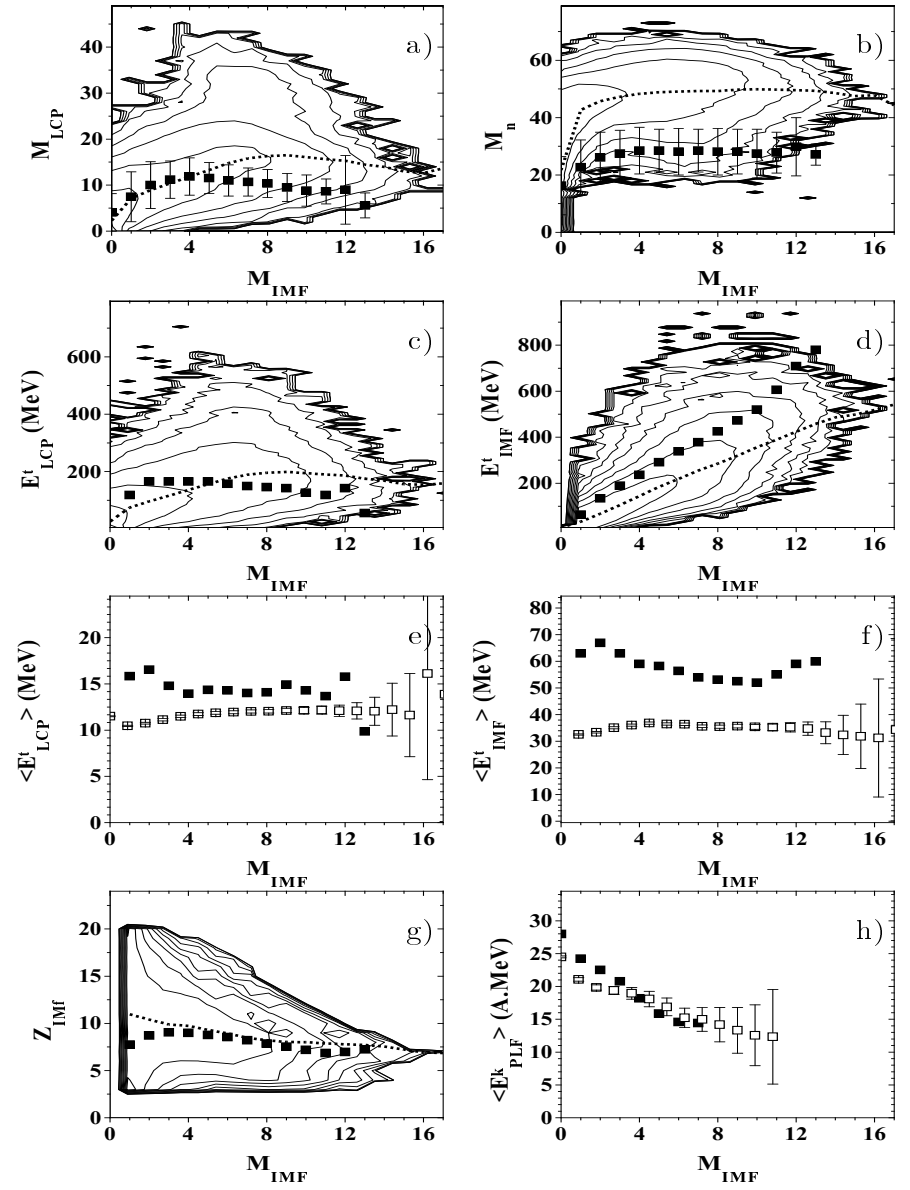

Figure 15: Comparison of measured observables [th to the results of calculations for the reaction ${ }^{136} \mathrm{Xe}+{ }^{209} \mathrm{Bi}$ at $28 \mathrm{~A} \mathrm{MeV}$. Solid symbols - experimental mean values for given IMF multiplicity, open symbols or thick dashed lines - calculated mean values, contour plots - calculated distributions. (a),(b) - dependencies of LCP and neutron multiplicity on IMF multiplicity, (c),(d) - total transverse energies of LCPs and IMFs, (e),(f) - mean transverse energies of LCPs and IMFs, (g) - IMF charge distributions, (h) - PLF kinetic energy. 
and dashed line - calculated values at $11^{\circ}$ and $40^{\circ}$ respectively, horizontal dotted line - unity ). As one can see the experimental yield ratios vary in much wider range than the calculated ones. The calculation typically underpredicts the yield ratios where yields of stable isotopes are compared to the yields of proton-rich isotopes. On the other hand, the yield ratios comparing the yield of neutron-rich isotope ${ }^{10} \mathrm{Be}$ to the yields of less neutron-rich isotopes are overpredicted. This situation is demonstrated clearly in Fig. 14c where both isobaric and isotopic yield ratios are plotted against average N/Z-ratio of a pair of nuclei ( the vertical dotted line shows average N/Z-ratio of the stable isotopes with $\mathrm{Z}=3-6$ ). Thus, the production of both proton- and neutron-rich isotopes seems to be overpredicted in the calculation when compared to the yields of stable isotopes. Similar situation was observed also in the original work when using both QMD-SMM and BUU-SMM calculations. Since the slope of plots in Fig. 14a,b can be related to temperature, it seems that the isotopic composition of fragments is determined at lower temperature than it is assumed in the SMM calculation. A possible explanation can be the collective flow of particles induced in the most violent phase of the collision. The inconsistencies in description of de-excitation in the SMM code can be possibly ruled out since it performs reasonably well in the case where hot quasiprojectiles have been created in the peripheral collisions of ${ }^{28} \mathrm{Si}$ beam with ${ }^{112,124} \mathrm{Sn}$ targets at 30 and 50 $\mathrm{A} \mathrm{MeV} \mathrm{[2,} \mathrm{35].} \mathrm{There} \mathrm{the} \mathrm{mechanism} \mathrm{of} \mathrm{quasiprojectile} \mathrm{production} \mathrm{is} \mathrm{described} \mathrm{well} \mathrm{using} \mathrm{DIT-}$ code of Tassan-Got [1] while the SMM code 34] describes reasonably well the de-excitation. The simulation employing both codes gives good description of the isobaric ratio $\mathrm{Y}\left({ }^{3} \mathrm{H}\right) / \mathrm{Y}\left({ }^{3} \mathrm{He}\right)[35$ ]. In such a reaction the collective flow apparently does not play a role. In the case of reaction ${ }^{58} \mathrm{Fe}+{ }^{58} \mathrm{Ni}$ at $30 \mathrm{~A} \mathrm{MeV}$ one can attribute the inconsistencies in description of isotopic and isobaric ratios at central angles to non-thermal effects rather than to a breakdown of description of de-excitation by SMM code at transition from masses 20-30 to 60-90. The kinetic energy of a collective flow can not be included into thermal energy and the temperature at the freeze-out becomes lower. In any case, the isotopic distributions seem to provide detailed insight into reaction dynamics. The calculation presented here is able to describe the inclusive characteristics like spectra and charge distributions at different angles and provide correct overall characteristics of the hot source. Nevertheless, additional assumptions concerning the amount of energy transformed into thermal degrees of freedom are necessary ( the same applies also to QMD-SMM and BUU-SMM calculations presented in the original work [33] ). Further studies are necessary in this direction.

In a recent experimental work Toke et al. [4] reported the observation of a new dynamical mechanism of fragment emission. The reaction ${ }^{136} \mathrm{Xe}+{ }^{209} \mathrm{Bi}$ at $28 \mathrm{~A} \mathrm{MeV}$ was studied in virtually $4 \pi$-geometry. Several experimental observables have been studied as a function of the multiplicity of intermediate mass fragments with $\mathrm{Z}=3$ - 20. Independence of the charge and transverse energy of IMFs, binary character of the reaction, simultaneous saturation of the multiplicity of neutrons and light charged particles ( saturation of heat content ) and absence of the competition between IMF emission and thermal emission have been interpreted as the main evidences of a new process where intermediate mass fragments are emitted dynamically without competition with emission of light particles. The IMFs in the events with IMF multiplicity 2 and 4 were emitted dominantly at parallel velocities between 0 and $5 \mathrm{~cm} / \mathrm{ns}$ in the c.m. frame.

The experimental results have been compared to the results of the calculation. As in the previous case the SMM code was used for de-excitation. Both sources have been de-excited independently. Results are given in the Fig. 15. For realistic comparison the calculated IMF multiplicities have been corrected to the geometric coverage of the detector setup which was $90 \%$ [36]. As one can see the calculation reproduces the simultaneous saturation of neutron and LCP multiplicity ( Fig. 15a,b ). The absolute values of multiplicity in the saturation region are overestimated for LCPs even when taking into account geometric coverage of the experimental setup ( in the case of LCPs 
the overall detection efficiency can be estimated to be close to total geometrical coverage ). In the case of neutrons the detection efficiency was estimated to be close to $70 \%$ [36] what brings the calculated neutron multiplicities just to the upper experimental limit. The trends of total transverse energies are tracked reasonably well for both LCPs and IMFs ( Fig. 15c,d ). The experimental transverse energies of both IMFs and LCPs are underpredicted by the calculation by a practically constant amount of energy for a wide range of IMF multiplicities ( Fig. 15, f ) and the experimental IMF charge and energy of projectile-like fragment are reproduced well for IMF multiplicities above 4 which can be attributed to violent collisions ( Fig. 15g,h ). For lower IMF multiplicities where peripheral collisions dominate there are inconsistencies ( Fig. 15e,f,g,h ) which can be attributed in part to the inconsistencies in the transition region observed also in other reactions. Also the geometric restrictions of the experimental setup at forward angles not taken into account in the calculations can be a source of inconsistencies. The increase of the IMF transverse energy at highest experimental multiplicities ( Fig. 15d,f) is based on data points with very low statistics.
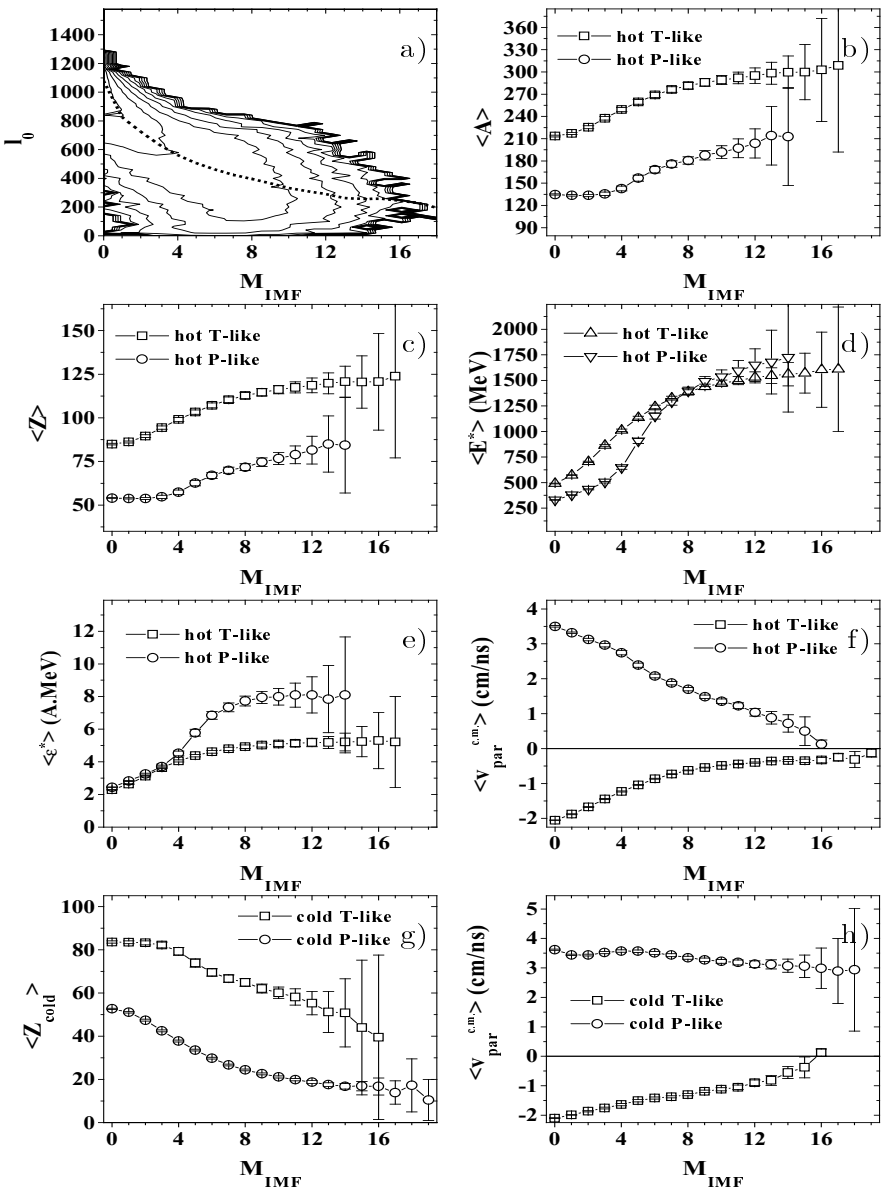

Figure 16: Calculated observables for the reaction ${ }^{136} \mathrm{Xe}+{ }^{209} \mathrm{Bi}$ at $28 \mathrm{~A} \mathrm{MeV}$. (a) - distribution of initial angular momenta ( thick dashed line - mean values for given IMF multiplicity), (b) - (h) - various calculated characteristics of both projectile- and target-like hot and cold sources.

As one can see on Fig. 15 the calculation reproduces the overall trends of the experimental data which have been interpreted as an evidence of dynamical emission of IMFs. The effect which was observed experimentally appears to be caused by a gradual increase of the mass and excitation 
energy of the emitting source with increasing centrality. Various calculated characteristics of both projectile- and target-like hot and cold sources are given in Fig. 16. As one can see the mass and charge of the hot target-like source ( which is produced in violent collisions with the probability exceeding $90 \%$ ) reach values up to 300 ( Fig. 16b ) and 120 ( Fig. 16c ), respectively, while excitation energy reaches value $5 \mathrm{~A} \mathrm{MeV}$ ( Fig. 16d ). For such a source, relatively small change in the mass, charge and excitation energy can lead to opening or closing of various emission channels. For example, the inconsistency in the LCP saturation multiplicity observed in Fig. 15a can be corrected by subtracting $0.5 \mathrm{~A} \mathrm{MeV}$ from the excitation energy. This, in analogy to previous reactions suggests that the nominal excitation energy of the hot source is not fully transferred into thermal excitation energy which determines the properties of the fragment partition at the freezeout. Part of the source energy is transferred into kinetic energy of collective motion resulting in the non-thermal flow. In the particular case of the reaction ${ }^{136} \mathrm{Xe}+{ }^{209} \mathrm{Bi}$ at $28 \mathrm{~A} \mathrm{MeV}$ the collective flow energy can be roughly estimated by above mentioned value $0.5 \mathrm{~A} \mathrm{MeV}$. Such a non-thermal flow is also suggested by observed excess of experimental transverse energy of LCPs and IMFs when compared to calculation ( Fig. 15e,f ). There the collective flow energy can be estimated to approximately $3 \mathrm{MeV}$ per fragment charge unit leading to the value of about $1.2 \mathrm{~A} \mathrm{MeV}$ for transverse direction. Thus the collective flow energy of LCPs and IMFs in the transverse direction appears to be larger than the overall decrease of excitation energy due to flow. It is of interest for further studies to investigate if it is caused by anisotropic profile of collective flow energy or if it coincides with lowering of transverse energy of other reaction products.

Parallel velocity of the emitted fragments can be related to the calculated parallel velocities of both hot and cold projectile- and target-like sources ( Fig. 16f,h ). In the dominant scenario the hot target-like source moves at parallel velocity slightly below c.m. velocity while the cold projectile-like source has parallel velocity $3-4 \mathrm{~cm} / \mathrm{ns}$. According to the experimental paper [4] the IMFs in mostly peripheral collisions with IMF multiplicity 2 and 4 are emitted predominantly in the forward direction in the c.m. frame. Calculated parallel velocities for such collisions imply that the IMF angular distribution is strongly influenced by the Coulomb field of the cold fragment ( Fig. 16 ) which causes IMFs to be emitted with highest probability in the configuration between hot and cold source which has the lowest Coulomb energy. Such interaction can be the cause of higher experimental LCP and IMF transverse energies in peripheral collisions ( Fig. 15e,f ).

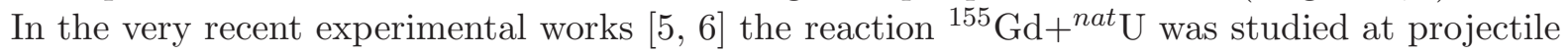
energy $36 \mathrm{~A} \mathrm{MeV}$. The experiment was carried out with geometric coverage close to $4 \pi$. In order to select the most central single-source events, a detailed event shape analysis was carried out. The events have been classified using the plot of total kinetic energy ( TKE ) vs. flow angle $\theta_{\text {flow }}$. Flow angle was calculated for each event using its sphericity and coplanarity. An observable $\Omega$ with values from 0 . to 1 . was defined in the most populated regions of the plot as a measure of centrality of the event. In the present work, the experimental TKE/ $\theta_{\text {flow }}$-plot was compared to the results of model calculation. As in previous cases, the SMM code 34] was used as afterburner and both sources have been de-excited independently. For each event a shape analysis was performed, sphericity and coplanarity were calculated and the flow angle was determined. Calculated TKE/ $\theta_{\text {flow }}$-plot is shown in Fig. 17 as a contour plot while solid squares give experimental points with $\Omega=0 ., 0.1$, ... , 1.0. Total kinetic energy is expressed relative to the available c.m. energy. As one can see the calculation is consistent with the experimental points. This allows to obtain dependencies of various calculated observables on $\Omega$.

In Fig. 18 are given multiplicities of various reaction products as a function of $\Omega$. Solid symbols represent the measured multiplicities of all charged particles, light charged particles, intermediate mass fragments and fragments with $\mathrm{Z} \geq 5$. Open symbols show calculated quantities. Both 


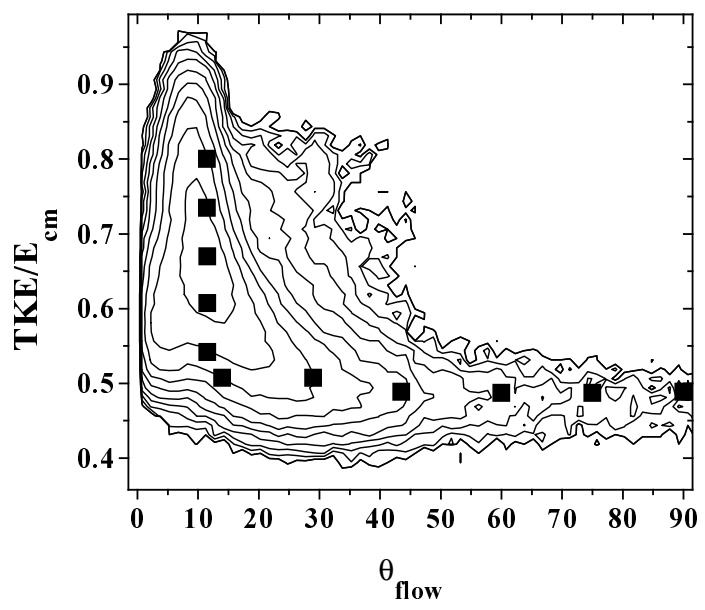

Figure 17: Total kinetic energy plotted versus flow angle $\theta_{\text {flow }}$ for the reaction ${ }^{155} \mathrm{Gd}+{ }^{\text {nat }} \mathrm{U}$ at $36 \mathrm{~A} \mathrm{MeV}$. Calculated TKE $/ \theta_{\text {flow }}$-plot is shown as contour plot while solid squares show experimental points [5] with $\Omega=0$., $0.1, \ldots, 1.0$. Total kinetic energy is expressed relative to the available c.m. energy.

experimental and calculated multiplicities exhibit saturation for $\Omega \geq 0.5$. Calculated saturation multiplicities typically overestimate experimental values.

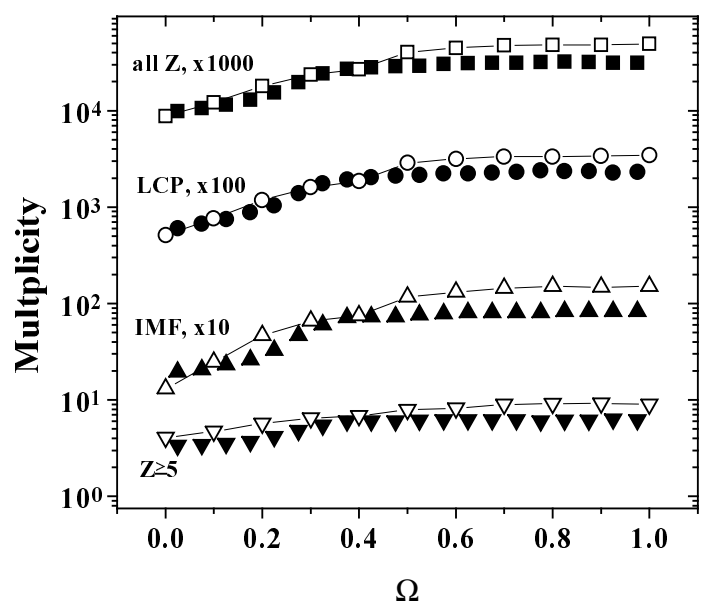

Figure 18: Multiplicities of various reaction products plotted as a function of $\Omega$ for the reaction ${ }^{155} \mathrm{Gd}+{ }^{\text {nat }} \mathrm{U}$ at $36 \mathrm{~A} \mathrm{MeV}$. Solid symbols - measured multiplicities [n] of all charged particles ( squares ), light charged particles ( circles ), intermediate mass fragments ( up triangles ) and fragments with $\mathrm{Z} \geq 5$ ( down triangles ). Open symbols calculated multiplicities for corresponding subsets of reaction products.

In a similar way to the reaction ${ }^{136} \mathrm{Xe}+{ }^{209} \mathrm{Bi}$ the inconsistency in multiplicities can be corrected by removing part of the excitation energy. In the present case in the most central collisions the calculated mass, charge and excitation energy of the hot source are $\mathrm{A}=386, \mathrm{Z}=153$ and $\epsilon^{*}=6.35$ $\mathrm{A} \mathrm{MeV}$. In this case, both target- and projectile-like source contribute because their excitation energy exceeds 3.5 A MeV what makes the event look like single-source event. Such events have been experimentally observed and their mass, charge and excitation energy have been determined using the LCP calorimetry method [6] ( $\mathrm{A}_{\text {exp }}=378, \mathrm{Z}_{\text {exp }}=150$ and $\left.\epsilon^{*}{ }_{\text {exp }}=6.5 \mathrm{~A} \mathrm{MeV}\right)$. Using the calculated properties of the single-source the observed saturation multiplicities can be reproduced 
with excitation energy being decreased by $1 \mathrm{~A} \mathrm{MeV} \mathrm{(} \mathrm{detection} \mathrm{efficiency} \mathrm{of} \mathrm{about} 90 \%$ was assumed ). This can be considered as an estimate of the energy of the collective flow which is consistent with estimate from experimental work $0.5 \pm 0.5 \mathrm{~A} \mathrm{MeV}$ obtained using the c.m. fragment kinetic energy spectra.

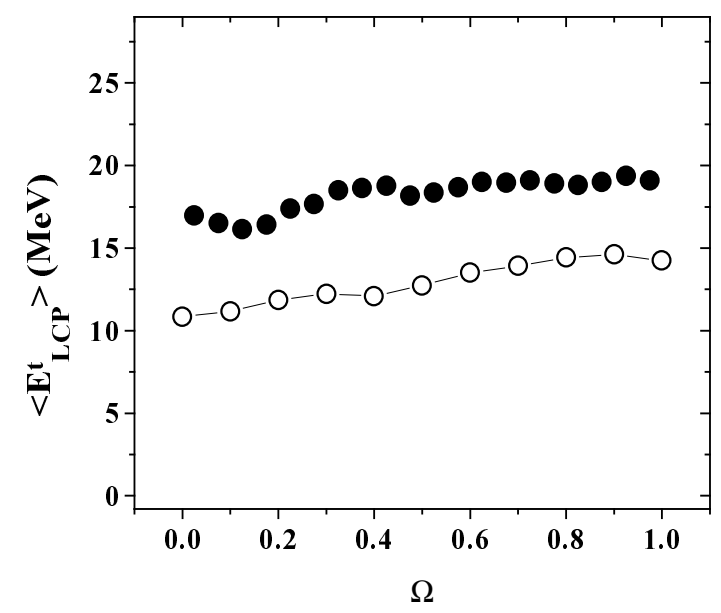

Figure 19: Mean transverse energy of light charged particles depending on $\Omega$ for the reaction ${ }^{155} \mathrm{Gd}+{ }^{\text {nat }} \mathrm{U}$ at 36 A MeV. Solid symbols - experiment []], open symbols - calculation.

Further estimate of the collective flow energy can be obtained from mean transverse energy of light charged particles which is shown in Fig. 19 as a function of $\Omega$. In analogy to the reaction ${ }^{136} \mathrm{Xe}+{ }^{209} \mathrm{Bi}$ the calculation underestimates the mean values of transverse energy by the same amount ( close to $5 \mathrm{MeV}$ ) for a wide range of events with different centrality. The kinetic energy of the collective flow can be in this case estimated as $3.5 \mathrm{MeV}$ per charge leading to flow energy approximately $1.4 \mathrm{~A} \mathrm{MeV}$. Thus, again in analogy to the reaction ${ }^{136} \mathrm{Xe}+{ }^{209} \mathrm{Bi}$, the energy of the collective flow of the light charged particles in the transverse direction is larger than the value of excitation energy to be subtracted in order to reproduce experimental multiplicities.

The experimental work [6] further provides the multiplicities of pre-equilibrium ( direct ) particles emitted in coincidence with single-source events. For the events with $\theta_{\text {flow }}>70^{\circ}$ typically 7 preequilibrium emissions occurred ( 3 neutrons and 4 LCPs ). In the calculation, mean pre-equilibrium multiplicity accompanying single-source events is $6.5 \pm 0.5\left(\mathrm{M}_{n}^{p r e}=3.8 \pm 0.4\right.$ and $\left.\mathrm{M}_{L C P}^{\text {pre }}=2.7 \pm 0.3\right)$. There is reasonable agreement in overall multiplicity of pre-equilibrium emissions while the larger multiplicity of neutrons in the calculation can be possibly explained by more LCP emission channels in the experimental data $(\mathrm{p}, \mathrm{d}, \mathrm{t}, \alpha)$ than in the calculation $(\mathrm{p}, \alpha)$.

Another heavy system studied recently in $4 \pi$-geometry was ${ }^{197} \mathrm{Au}+{ }^{197} \mathrm{Au}$ at projectile energy $35 \mathrm{~A} \mathrm{MeV} \mathrm{[7,} \mathrm{8].} \mathrm{For} \mathrm{this} \mathrm{symmetric} \mathrm{heavy} \mathrm{system} \mathrm{an} \mathrm{analysis} \mathrm{of} \mathrm{central} \mathrm{collisions} \mathrm{was} \mathrm{performed}$ with emphasis put on the properties of emitted fragments. For charge distributions measured in the most central collisions a model analysis was carried out using SMM code and mass, charge, excitation energy and flow energy of the single-source have been determined. Two sets of source parameters have been determined [8] which reproduced the data equally well - $\mathrm{A}=343, \mathrm{Z}=138$, $\epsilon^{*}=6.0 \mathrm{~A} \mathrm{MeV}$ ( flow energy was 0 . and freeze-out density was set $\rho_{0} / 3$ ) and $\mathrm{A}=315, \mathrm{Z}=126$, $\epsilon^{*}=4.8 \mathrm{~A} \mathrm{MeV}$ ( flow energy was $0.8 \mathrm{~A} \mathrm{MeV}$ and freeze-out density was set $\rho_{0} / 6$ ). Calculation analogous to previous cases gives for most central collisions $\left(l_{0} \leq 100\right)$ a hot source with mean parameters $\mathrm{A}=341, \mathrm{Z}=136, \epsilon^{*}=6.45 \mathrm{~A} \mathrm{MeV}$. Corresponding cold source still exists $(\mathrm{A}=$ $52, \mathrm{Z}=22, \mathrm{E}^{*}=110 \mathrm{MeV}$ ) but de-excites mostly by neutron emission. The properties of the 
calculated source are in reasonable agreement with the heavier of the sources. In a similar way to previous reactions an estimate of thermal excitation energy at freeze-out was obtained using mean IMF multiplicity. In the most central collision of ${ }^{197} \mathrm{Au}+{ }^{197} \mathrm{Au}$ at $35 \mathrm{~A} \mathrm{MeV}$ the experimentally determined mean IMF multiplicity was $10.8 \pm 1$. Calculation reproduces this value when subtracting $0.75 \mathrm{~A} \mathrm{MeV}$ (detection efficiency of $90 \%$ was assumed ). Such a value of collective non-thermal energy is comparable with previous cases. The difference in the amount of collective flow between SMM calculations used in [8] and here can originate in the freeze-out hypothesis used. The SMM calculation used in [8] used a fixed freeze-out density that leads to fixed Coulomb barrier and higher sensitivity of mean c.m. kinetic energy to freeze-out temperature. In the present work the freeze-out density is determined according to prescription depending on fragment multiplicity [34] in which freeze-out density ( and thus Coulomb barrier ) decrease with increasing fragment number thus leading to weaker sensitivity. The experimental mean fragment c.m. kinetic energies for most central collisions given in [8] can not be used in order to distinguish between the two hypotheses because of large uncertainties caused by low experimental statistics.

In summary, the investigations presented in this section demonstrate that the model calculation provides consistent description of the production mechanism of the hot source which further undergoes multifragmentation. The calculation described the overall properties of the hot source like mass, charge and available c.m. energy for wide range of experimental data, especially well for the highly complete and model independent data obtained in $4 \pi$-geometry. Thermodynamical properties of the source determined from fragment partitions suggest that the energy available in the source frame is not fully transferred into heat and a collective flow starts to play role. This is indicated indirectly by several observables such as particle multiplicities, transverse energies and isotopic/isobaric ratios. In any case, the systematic trend rather than any particular case can be considered as an indication of the non-thermal flow. Further detailed studies on that subject possibly by investigating large set of observables in the same system would be of great interest. The estimated energy of the collective flow does not seem to differ dramatically for various systems and the values extracted are in good agreement with other studies ( e.g. [37] and references therein ). Furthermore, the collective flow energy seems to be practically constant for events with different centralities. Such a behavior can be possibly explained within the present physical picture by the interplay of two different effects. In central collisions, the participant zone increases rapidly and compressional effects should lead to a flow concentrated in transverse direction. In peripheral collisions, the charge of the cold spectator is significant enough to focus the fragments emitted in the mid-velocity region into transverse direction. Such an enhancement of fragment multiplicity at central angles was described e.g. in work [38].

\section{Possible limitations of the model}

With increasing projectile energy, the production of the spectator-participant-like three-body events should start to play an important role. As primary candidates for three-body events can be considered the events where the relative motion of the participant zone and the capturing spectator leads to values of intrinsic angular momenta above the critical angular momentum for fusion. Furthermore, at projectile energies much above the Fermi energy the intra-nuclear cascade should take place and change the properties of the pre-equilibrium source. For projectile energies below 20 $\mathrm{A} \mathrm{MeV}$ the nucleus-nucleus interaction becomes more complex because of the effect of proximity potential. The sum-rule model [39] of Wilczynski et al. employing a concept of angular momentum windows determined by critical angular momenta for different incomplete fusion channels can be used in this energy region. 


\section{Conclusions}

The comparisons to the wide range of experimental observables measured in various reactions in the Fermi energy domain appear to imply that the present approach describes consistently the main features of the violent processes leading to the production of excited projectile-like, mid-velocity and fusion-like sources in the projectile energy range between 20 and $100 \mathrm{~A} \mathrm{MeV}$. Different stages of the collision can be distinguished and related to each other using a simple phenomenological assumptions based on a geometrical picture of the collision. The mean multiplicity of pre-equilibrium particles is described satisfactory by using phenomenological approach employing an exciton concept. Furthermore, a direct relation of the mean multiplicity of pre-equilibrium particles to the geometrical aspects of the reaction is demonstrated. The pre-equilibrium emission appears to be a consequence of the radial motion of the projectile-target system and ceases when radial motion is transferred into tangential via Coulomb interaction. At that point, according to the degree of tangential motion a deep-inelastic transfer or a formation of the participant and spectator zones follows. The participant zone can be captured by one of the spectator zones and incomplete fusion occurs. The Coulomb interaction between the projectile and target as a whole plays still an important role in such a violent reaction scenario. The resulting motion along the classical Coulomb trajectories furthermore assures conservation of angular momentum. Concerning the applicability of the model, it appears to describe consistently in many projectile-target systems the mass, charge and excitation energy of hot nuclei which later undergo multifragmentation. Furthermore, the presence of non-thermal effects can be expected from observed multiplicities, transverse energies and isotopic/isobaric yield ratios. The values of flow energy are similar for different systems and are practically constant within one projectile-target system for collisions with different centrality.

The author would like to thank G.A. Souliotis and S.J. Yennello for the support and fruitful and

stimulating discussions. Furthermore, the author would like to thank A. Sanzhur for discussions concerning pre-equilibrium emission. The author would also like to thank F.-P. Hessberger, D.D. Bogdanov, B.I. Pustylnik and A.V. Yeremin for many valuable discussions on low energy reaction mechanisms which helped to formulate some of the basic concepts of this work. Finally, the author would like to thank L. Tassan-Got for the use of his DIT code, R.J. Charity for the use of the code GEMINI and A.S. Botvina for the use of the SMM code. This work was supported in part by the Robert A. Welch Foundation through grant No. A-1266, the Department of Energy through grant No. DE-FG03-93ER40773, and through grant VEGA-2/1132/21.

\section{References}

[1] L. Tassan-Got, C. Stéfan, Nucl. Phys A 524 (1991) 121.

[2] M. Veselsky, R.W. Ibbotson, R. Laforest, E. Ramakrishnan, D.J. Rowland, A. Ruangma, E.M. Winchester, E. Martin, S.J. Yennello, Phys. Rev. C 62 (2000) 064613.

[3] D. Dore, Ph. Buchet, J.L. Charvet, R. Dayras, L. Nalpas, D. Cussol, T. Lefort, R. Legrain, C. Volant, G. Auger, Ch.O. Bacri, N. Bellaize, F. Bocage, R. Bougault, B. Bouriquet, R. Brou, A. Chbihi, J. Colin, A. Demeyer, D. Durand, J.D. Frankland, E. Galichet, E. Genouin-Duhamel, E. Gerlic, D. Guinet, S. Hudan, P. Lautesse, F. Lavaud, J.L. Laville, J.F. Lecolley, C. Leduc, N. Le Neindre, O. Lopez, M. Louvel, A.M. Maskay, J. Normand, M. Parlog, P. Pawlowski, E. Plagnol, M.F. Rivet, E. Rosato, F. Saint-Laurent, J.C. Steckmeyer, M. Stern, G. Tabacaru, B. Tamain, L. Tassan-Got, O. Tirel, E. Vient, J.P. Wieleczko, Phys. Lett. B 491 (2000) 15. 
[4] J. Toke, D.K. Agnihotri, S.P. Baldwin, B. Djerroud, B. Lott, B.M. Quednau, W. Skulski, W.U. Schroder, L.G. Sobotka, R.J. Charity, D.G. Sarantites, R.T. de Souza, Phys. Rev. Lett. 77 (1996) 3514.

[5] J.D. Frankland, Ch.O. Bacri, B. Borderie, M.F. Rivet, M. Squalli, G. Auger, N. Bellaize, F. Bocage, R. Bougault, R. Brou, Ph. Buchet, A. Chbihi, J. Colin, D. Cussol, R. Dayras, A. Demeyer, D. Dorr, D. Durand, E. Galichet, E. Genouin-Duhamel, E. Gerlic, D. Guinet, Ph. Lautesse, J.L. Laville, J.F. Lecolley, R. Legrain, N. Le Neindre, O. Lopez, M. Louvel, A.M. Maskay, L. Nalpas, A.D. Nguyen, M. Parlog, J. Prter, E. Plagnol, E. Rosato, F. Saint-Laurent, S. Salou, J.C. Steckmeyer, M. Stern, G. Tabacaru, B. Tamain, O. Tirel, L. Tassan-Got, E. Vient, C. Volant, J.P. Wieleczko, Nucl. Phys. A 689, (2001) 905.

[6] J.D. Frankland, Ch.O. Bacri, B. Borderie, M.F. Rivet, M. Squalli, G. Auger, N. Bellaize, F. Bocage, R. Bougault, R. Brou, Ph. Buchet, A. Chbihi, J. Colin, D. Cussol, R. Dayras, A. Demeyer, D. Dore, D. Durand, E. Galichet, E. Genouin-Duhamel, E. Gerlic, D. Guinet, Ph. Lautesse, J.L. Laville, J.F. Lecolley, R. Legrain, N. Le Neindre, O. Lopez, M. Louvel, A.M. Maskay, L. Nalpas, A.D. Nguyen, M. Parlog, J. Peter, E. Plagnol, E. Rosato, F. Saint-Laurent, S. Salou, J.C. Steckmeyer, M. Stern, G. Tabacaru, B. Tamain, O. Tirel, L. Tassan-Got, E. Vient, C. Volant, J.P. Wieleczko, Nucl. Phys. A 689, (2001) 940.

[7] M. D’Agostino, G.J. Kunde, P.M. Milazzo, J.D. Dinius, M. Bruno, N. Colonna, M.L. Fiandri, C.K. Gelbke, T. Glasmacher, F. Gramegna, D.O. Handzy, W.C. Hsi, M. Huang, M.A. Lisa, W.G. Lynch, P.F. Mastinu, C.P. Montoya, A. Moroni, G.F. Peaslee, L. Phair, R. Rui, C. Schwarz, M.B. Tsang, G. Vannini, C. Williams, Phys. Rev. Lett. 75 (1995) 4373.

[8] M. D’Agostino, A.S. Botvina, P.M. Milazzo, M. Bruno, G.J. Kunde, D.R. Bowman, L. Celano, N. Colonna, J.D. Dinius, A. Ferrero, M.L. Fiandri, C.K. Gelbke, T. Glasmacher, F. Gramegna, D.O. Handzy, D. Horn, W.C. Hsi, M. Huang, I. Iori, M.A. Lisa, W.G. Lynch, L. Manduci, G.V. Margagliotti, P.F. Mastinu, I.N. Mishustin, C.P. Montoya, A. Moroni, G.F. Peaslee, F. Petruzzelli, L. Phair, R. Rui, C. Schwarz, M.B. Tsang, G. Vannini, C. Williams, Phys. Lett. B 371 (1996) 175.

[9] J.J. Griffin, Phys. Rev. Lett. 17 (1966) 478.

[10] J. Randrup, R. Vandenbosch, Nucl. Phys. A 474 (1987) 219.

[11] M. Veselsky, S. Saro, F.P. Heßberger, V. Ninov, S. Hofmann, D. Ackermann, Z. Phys. A 356 (1997) 403.

[12] M. Böhning, Nucl. Phys. A 152 (1970) 529.

[13] H. Fuchs, K. Möhring, Rep. Prog. Phys. 57 (1994) 231.

[14] C.K. Gelbke, D.H. Boal, Prog. Part. Nucl. Phys. 19 (1987) 33.

[15] J. Gosset, H.H. Gutbrod, W.G. Meyer, A.M. Poskanzer, A. Sandoval, R. Stock, G.D. Westfall, Phys. Rev. C 16 (1977) 629.

[16] R. Dayras, A. Pagano, J. Barrette, B. Berthier, D.M. De Castro Rizzo, E. Chavez, O. Cisse, R. Legrain, M.C. Mermaz, E.C. Pollacco, H. Delagrange, W. Mittig, B. Heusch, R. Coniglione, G. Lanzano, A. Palmeri, Nucl. Phys. A 460 (1986) 299. 
[17] A.Y. Abul-Magd, Z. Phys. A 298 (1980) 143.

[18] B.G. Harvey, M.J. Murphy, Phys. Lett. B 130 (1983) 373.

[19] W.A. Friedman, Phys. Rev. C 27 (1983) 569.

[20] J.-J. Gaimard, K.-H. Schmidt, Nucl. Phys. A 531 (1991) 709.

[21] N. Matsuoka, A. Shimizu, K. Hosono, T. Saito, M. Kondo, H. Sakaguchi, Y. Toba, A. Goto, F. Ohtani, N. Nakanishi, Nucl. Phys. A 311 (1978) 173.

[22] D.K. Agnihotri, B.Djerroud, J.Toke, W. Skulski, W.U. Schroder, B. Davin, E. Cornell, and R.T. De Souza, Adv. Nucl. Dyn. 1997, 3, 67.

[23] E. Holub, D. Hilscher, G. Ingold, U. Jahnke, H. Orf, H. Rossner, Phys. Rev. C 28 (1983) 252.

[24] D. Hilscher, Nucl. Phys. A 471 (1987) 77c.

[25] G. Casini, M. Bini, S. Calamai, R. Laforest, P.R. Maurenzig, A. Olmi, G. Pasquali, S. Piantelli, G. Poggi, F. Saint-Laurent, J.C. Steckmeyer, A.A. Stefanini, N. Taccetti, Eur. Phys. J. A 9 (2000) 491.

[26] J. Randrup, Nucl. Phys. A 307 (1978) 319; Nucl. Phys. A 327 (1979) 490; Nucl. Phys. A 383 (1982) 468.

[27] G.A. Souliotis, K. Hanold, W. Loveland, D.J. Morrissey, G.J. Wozniak, in Nuclear Chem. Ann. Report 1996-97, Oregon State University, 1997, http://www.orst.edu/dept/nchem/project/hvyrdu22.htm.

[28] K. Summerer, W. Bruchle, D.J. Morrissey, M. Schadel, B. Szweryn, W. Yang, Phys. Rev. C 42 (1990) 2546.

[29] R.J. Charity, M.A. McMahan, G.J. Wozniak, R.J. McDonald, L.G. Moretto, D.G. Sarantites, L.G. Sobotka, G. Guarino, A. Pantaleo, L. Fiore, A. Gobbi, K.D. Hildenbrand, Nucl. Phys. A 483 (1988) 371.

[30] G.A. Souliotis, K. Hanold, W. Loveland, D.J. Morrissey, G.J. Wozniak, submitted to Nucl. Phys. A.

[31] K. Hagel, R. Wada, J. Cibor, M. Lunardon, N. Marie, R. Alfaro, W. Shen, B. Xiao, Y. Zhao, Z. Majka, J. Li, P. Staszel, B.-A. Li, M. Murray, T. Keutgen, A. Bonasera, J. B. Natowitz, Phys. Rev. C 62 (2000) 034607.

[32] J. Lukasik, Z. Majka, Acta Phys. Pol. B 24 (1993) 1959.

[33] E. Ramakrishnan, H. Johnston, F. Gimeno-Nogues, D.J. Rowland, R. Laforest, Y-W. Lui, S. Ferro, S. Vasal, S. J. Yennello, Phys. Rev. C 57 (1998) 1803.

[34] J.P. Bondorf, A.S. Botvina, A.S. Iljinov, I.N. Mishustin, K. Sneppen, Phys. Rep. 257 (1995) 133.

[35] M. Veselsky, R.W. Ibbotson, R. Laforest, E. Ramakrishnan, D.J. Rowland, A. Ruangma, E.M. Winchester, E. Martin, S.J. Yennello, Phys. Rev. C 62 (2000) 41605(R). 
[36] J. Toke, B. Lott, S.P. Baldwin, B.M. Quednau, W.U. Schroder, L.G. Sobotka, J. Barreto, R.J. Charity, D.G. Sarantites, D.W. Stracener, R.T. de Souza, Phys. Rev. Lett. 75 (1995) 2920.

[37] P. Desesquelles, M. D’Agostino, A.S. Botvina, M. Bruno, N. Colonna, A. Ferrero, M.L. Fiandri, E. Fuschini, F. Gramegna, I. Iori, G.V. Margagliotti, P.F. Mastinu, P.M. Milazzo, A. Moroni, F. Petruzzelli, R. Rui, G. Vannini, J.D. Dinius, C.K. Gelbke, T. Glasmacher, D.O. Handzy, W.C. Hsi, M. Huang, M.A. Lisa, W.G. Lynch, C.P. Montoya, G.F. Peaslee, L. Phair, C. Schwarz, M.B. Tsang, C. Williams, Nucl. Phys. A 633 (1998) 547.

[38] A. S. Botvina, M. Bruno, M. D’Agostino, D.H.E. Gross, Phys. Rev. C 59 (1999) 3444.

[39] J. Wilczynski, K. Siwek-Wilczynska, J. van Driel, S. Gongrijp, D.C. Hageman, R.V. Janssens, J. Lukasiak, R.H. Siemssen, Phys. Rev. Lett. 45 (1980) 606. 